\title{
Derivation of effective models from heterogenous Cosserat media via periodic unfolding
}

\author{
Grigor Nika ${ }^{1}$
}

Received: 25 March 2021 / Revised: 8 June 2021 / Accepted: 16 June 2021

(c) The Author(s) 2021

\begin{abstract}
We derive two different effective models from a heterogeneous Cosserat continuum taking into account the Cosserat intrinsic length of the constituents. We pass to the limit using homogenization via periodic unfolding and in doing so we provide rigorous proof to the results introduced by Forest, Pradel, and Sab (Int. J. Solids Struct. 38 (2627): 4585-4608 '01). Depending on how different characteristic lengths of the domain scale with respect to the Cosserat intrinsic length, we obtain either an effective classical Cauchy continuum or an effective Cosserat continuum. Moreover, we provide some corrector type results for each case.
\end{abstract}

Keywords Cosserat continuum · Size effects · Homogenization · Periodic unfolding · Correctors

Mathematics Subject Classification 35A15 · 35Q74 · 74M25 · 74Q15

\section{Introduction}

In recent years it has been widely observed that mechanical properties of composite materials that are used in a variety of applications depend on different characteristic lengths. These characteristic lengths are determined either by the structure itself or by the characteristics of an underlying microstructure or by a combination of both [22-24,34,36]. The phenomena described above can give rise to corresponding macroscopic properties of the composite that may be vastly different from the underlying material properties. Moreover, in cases where these characteristic lengths of the problem become comparable with the characteristic length of the microstructure, classical theory of continuum mechanics loses its accuracy in describing the mechanical behav-

\footnotetext{
$凶$ Grigor Nika

grigor.nika@wias-berlin.de

1 Weierstrass Institute for Applied Analysis and Stochastics, Mohrenstraße 39, 10117 Berlin, Germany
} 
ior of such materials. These type of phenomena described above are often referred to as size effects and one way of accounting for size effects in composites is to model them using generalized continuum theories. One of the earliest generalized continuum theories was that of the Cosserat brothers [3] where they introduced the notion of the couple-stress. Their original development of the theory was largely underappreciated during their time only to be revisited again (and expanded) in the early sixties onwards [10-12,26-29,32,40,41]. Cosserat continuum mechanics incorporates size effects naturally through an intrinsic length scale parameter $\ell_{c}$ which, loosely speaking, can be considered as a measure of the absolute size of the constituents in the unit cell. Generalized continuum theories have applications in the modeling of materials with microstructure, granular materials, fibrous materials, or materials with a lattice structure [13,14,27,28,39]. One of the methods specifically designed for the analysis of highly heterogeneous and microstructured materials is the theory of homogenization $[1,2,30,38]$. In this theory, the effective material properties of periodic structures are defined by the analysis on a periodicity cell and, in turn, these properties depend on the mechanics of constituents and the geometry of the periodic structure but are independent of the external boundary conditions and applied forces. They are normally determined in the limit as the size of the microstructure $\varepsilon \rightarrow 0$. Moreover, homogenization seems like a natural fit to explore connections between generalized continuum theories and classical theory for heterogenous structures.

Generalized continuum theories fall into two categories: higher grade theory that introduces higher gradients of the displacement field to the usual strain tensor and higher order theory that includes additional degrees of freedom. Regarding the former there is a vast literature of deriving second grade models through constitutive modelling, dimensional reduction or homogenization techniques $[9,16,18,19,35,37,39]$. We point out that the authors in [9] provide a historical perspective and theoretical overview of higher grade continua. Regarding the latter, where one allows for additional degrees of freedom as in a Cosserat continuum, a series of works appeared in the late nineties addressing estimation of effective properties of heterogenous Cosserat materials taking into account size effects [13-15]. In particular, the authors in [13] consider periodic heterogenous Cosserat material taking into account a hierarchy of three characteristic lengths when obtaining the homogenous equivalent medium: the characteristic size of inhomogeneities, the Cosserat intrinsic length of the constituents, and the typical size of the considered structure. Heuristically, using two-scale expansions, the authors derived different homogenized models based on how the three characteristic lengths scale with respect to one another and, moreover, validated their results using finite element calculations.

The aim of this work is to provide the mathematical underpinnings that make the work in [13] mathematically rigorous. Specifically, we consider a periodic Cosserat body $\Omega$ with body forces and body-couples acting on it,

$$
\begin{array}{ll}
-\operatorname{div} \sigma=f & \text { in } \Omega, \\
-\operatorname{div} \mu-\epsilon: \sigma=g & \text { in } \Omega,
\end{array}
$$


with $\sigma$ the non-symmetric stress tensor, $\mu$ the couple-stress tensor, $\epsilon$ the Levi-Civita tensor with $\epsilon: \sigma=\sum_{j, k=1}^{3} \boldsymbol{\epsilon}_{i j k} \sigma_{j k}$ for $i \in\{1,2,3\}, \boldsymbol{f}$ are body forces, and $\boldsymbol{g}$ are body couples. Moreover, the constitutive relations are given by,

$$
\sigma_{j i}=\sum_{k, \ell=1}^{3}\left(C_{j i k \ell} \gamma_{k \ell}+B_{j i k \ell} \kappa_{k \ell}\right), \quad \mu_{j i}=\sum_{k, \ell=1}^{3}\left(B_{k \ell j i} \gamma_{k \ell}+L_{j i k \ell} \kappa_{k \ell}\right)
$$

for $\mathrm{i}, \mathrm{j} \in\{1,2,3\}$.

Here, we have defined the non-symmetric strain tensor and the torsion tensor (or curvature-twist tensor or curvature tensor or curvature) respectively, by,

$$
\gamma_{j i}:=\partial_{x_{j}} u_{i}-\sum_{k=1}^{3} \epsilon_{k j i} \varphi_{k} \quad \text { and } \quad \kappa_{j i}:=\partial_{x_{j}} \varphi_{i} \text { for } i, j \in\{1,2,3\}
$$

where $\boldsymbol{u}$ is the displacement and $\boldsymbol{\varphi}$ is the rotation. In this work we will assume we deal with centro-symmetric bodies and hence the fourth order tensor $B_{i j k \ell} \equiv 0$ for $i, j, k, \ell \in\{1,2,3\}$ [32]. Using the dimensional analysis done in [13] to obtain the hierarchy of models based on the scaling of the Cosserat intrinsic length $\ell_{c}$ with respect to the overall length of the domain $\underline{l}$ or the length of the periodic cell $\ell$, and the periodic unfolding method we pass to the limit in each case. We obtain two different effective models: If $\ell_{c} / \ell$ remains constant when $\ell / \underline{l}$ goes to zero we obtain an effective Cauchy continuum where the effective moduli tensor depends on a standard set of local problems as in classical homogenization and on a set of local problems that contain the contribution of the rotations. If $\ell_{c} / \underline{l}$ remains constant when $\ell / \underline{l}$ goes to zero then we obtain an effective Cosserat continuum. In both cases we verify the results in [13]. Additionally, we prove certain corrector type results using the adjoint of the unfolding operator (the averaging operator).

The paper is organized as follows: In Sect. 2 we reproduce the dimensional analysis in [13], provide some background, and set up the model. In Sect. 3 we recall the definition of the unfolding and averaging operators and prove the main results. Section 4 is devoted to proving certain corrector type results using the averaging operator. We need to remark that we refer to the above as corrector type results as they involve both the displacement and the rotations unlike in classical elasticity where only the displacement is involved. To the author's knowledge, these corrector type results are new in their entirety. Finally, in Sect. 5 we provide some conclusions and remarks.

\section{Background and set up of the problem}

\subsection{Cosserat intrinsic length of the constituents}

Let $\boldsymbol{l}$ be the characteristic length of the domain $\Omega$ and $\ell$ the characteristic length of the periodic cell. We define the dimensionless coordinates, displacement, and rotation as in [13], 


$$
x^{*}=\frac{x}{\underline{l}}, \quad u^{*}\left(x^{*}\right)=\frac{u(x)}{\underline{l}}, \quad \varphi^{*}\left(x^{*}\right)=\varphi(x) .
$$

In Cosserat media there is another length scale parameter that is of importance, namely, the Cosserat intrinsic length $\ell_{c}$ of the constituents $[13,14]$. The following nondimensionalization was done in $[13,14]$ and we include it here for completion of the presentation. Hence, in accordance with [13], the Cosserat intrinsic length is defined as follows,

$$
\mathcal{C}=\mathcal{L} \ell_{c}^{2}
$$

where $\mathcal{L}=\max _{z \in Y_{\ell}}\left|L_{j i k l}(z)\right|, \mathcal{C}=\max _{z \in Y_{\ell}}\left|C_{j i k l}(z)\right|$ for $i, j, k, l \in\{1,2,3\}$, and $Y_{\ell}=(-\ell / 2, \ell / 2]^{d}$ is periodic cell characterizing the body $\Omega$. Additionally, the nonsymmetric strain and curvature nondimensionalize respectively as,

$$
\gamma^{*}=\gamma \quad \text { and } \quad \kappa^{*}=\underline{\boldsymbol{l}} \kappa .
$$

Moreover, we define the nondimensional stress, couple-stress, and fourth order material tensors as follows,

$$
\begin{array}{ll}
\sigma^{*}=\mathcal{L}^{-1} \sigma, & \mathbb{L}^{*}\left(\boldsymbol{x}^{*}\right)=\mathcal{L}^{-1} \mathbb{L}(\boldsymbol{x}), \\
\mu^{*}=(\mathcal{L} \underline{l})^{-1} \mu, & \mathbb{C}^{*}\left(\boldsymbol{x}^{*}\right)=\mathcal{C}^{-1} \mathbb{C}(\boldsymbol{x})
\end{array}
$$

We remark that the fourth order material tensors $\mathbb{L}^{*}\left(\boldsymbol{x}^{*}\right)=\left\{L_{j i k \ell}^{*}(\boldsymbol{x} *)\right\}_{j, i, k, \ell=1}^{3}$ and $\mathbb{C}^{*}\left(\boldsymbol{x}^{*}\right)=\left\{C_{j i k \ell}^{*}(\boldsymbol{x} *)\right\}_{j, i, k, \ell=1}^{3}$ are $Y^{*}$ periodic where,

$$
Y^{*}=\frac{\ell}{\underline{l}} Y, \quad Y:=\left(-\frac{1}{2}, \frac{1}{2}\right]^{d}
$$

Hence, the system of Eq. in (1.1) scales as,

$$
\begin{array}{ll}
-\operatorname{div} \sigma^{*}=f^{*} & \text { in } \Omega, \\
-\operatorname{div} \mu^{*}-\epsilon: \sigma^{*}=g^{*} & \text { in } \Omega,
\end{array}
$$

where $f^{*}$, and $\boldsymbol{g}^{*}$ are the appropriately scaled body forces and body-couples (see [13, Eq. (14), pg. 4589]) and with constitutive laws,

$$
\sigma_{j i}^{*}=\sum_{k, \ell=1}^{3} C_{j i k \ell}^{*} \gamma_{k \ell}, \quad \mu_{j i}^{*}=\sum_{k, \ell=1}^{3}\left(\frac{\ell_{c}}{\underline{l}}\right)^{2} L_{j i k \ell}^{*} \kappa_{k \ell}^{*} \quad \text { for } i, j \in\{1,2,3\}
$$


Thus, one can generate an $\varepsilon$ periodic problem by defining the nondimensional number $\varepsilon$ as the ratio of $\ell / \underline{l}$ and let $\varepsilon \rightarrow 0$ to obtain an effective medium. However, different cases ought to be considered depending on how $\ell_{c}$ scales with $\ell$ and $\underline{l}$, respectively [13]. Here we consider the cases,

$$
\begin{aligned}
& \ell_{c} / \ell \sim 1, \\
& \ell_{c} / \underline{l} \sim 1 .
\end{aligned}
$$

If $\ell_{c} / \ell \sim 1$ then $\mu_{j i}^{*}=\sum_{k, \ell=1}^{3}\left(\frac{\ell_{c}}{\underline{l}}\right)^{2} L_{j i k \ell}^{*} \kappa_{k \ell}^{*}$ for $i, j \in\{1,2,3\}$. Using the definition $\varepsilon=\ell / \underline{l}$ and omitting the $*$ notation the couple stress becomes,

$$
\mu_{j i}^{\varepsilon}=\varepsilon^{2} \sum_{k, \ell=1}^{3} L_{j i k \ell}\left(\frac{\boldsymbol{x}}{\varepsilon}\right) \kappa_{k \ell}^{\varepsilon} .
$$

If $\ell_{c} / \underline{\boldsymbol{l}} \sim 1$ then $\mu_{j i}^{*}=\sum_{k, \ell=1}^{3}\left(\frac{\ell_{c}}{\underline{l}}\right)^{2} L_{j i k \ell}^{*} \kappa_{k \ell}^{*}$ for $i, j \in\{1,2,3\}$ becomes,

$$
\mu_{j i}^{\varepsilon}=\sum_{k, \ell=1}^{3} L_{j i k \ell}\left(\frac{\boldsymbol{x}}{\varepsilon}\right) \kappa_{k \ell}^{\varepsilon} .
$$

The former allows one to pass from a Cosserat continuum in the microscale to a Cauchy continuum in the macroscale as $\varepsilon \rightarrow 0$ where all the relevant information are now captured in a new homogenized tensor which can be computed explicitly with the aid of an additional set of local problems. The latter allows one to obtain an effective Cosserat medium as $\varepsilon \rightarrow 0$.

\section{Notation}

To expedite the presentation of our results, here onwards we will make use of the following notation:

- We employ the Einstein notation of repeated indices unless otherwise stated.

- The real numbers $\alpha, \beta \in \mathbb{R}$ are generic constants such that $0<\alpha$ and $0<\beta$.

- For any two second order tensors $\mathrm{A}$ and $\mathrm{B}$ we define the Frobenius inner product as $\mathrm{A}: \mathrm{B}=A_{i j} B_{i j}$.

- $\mathcal{M}_{d}^{4}(\alpha, \beta, \Omega)=\left\{\right.$ all fourth order tensors in $L^{\infty}\left(\Omega ; \mathbb{R}^{d \times d \times d \times d}\right)$ acting on matrices such that for any matrix $\zeta \in \mathbb{R}^{d \times d}, \mathbb{L}(\boldsymbol{x}) \zeta: \zeta \geq \alpha|\zeta|^{2}$ and $\beta|\zeta|^{2} \leq$ $\mathbb{L}^{-1}(\boldsymbol{x}) \zeta: \zeta$ for a.e. $\left.\boldsymbol{x} \in \Omega\right\}$.

- Any fourth order tensor of the form $\mathbb{T}^{\varepsilon}(\boldsymbol{x})$ is defined, as usual, by $\mathbb{T}^{\varepsilon}(\boldsymbol{x}):=\mathbb{T}\left(\frac{\boldsymbol{x}}{\varepsilon}\right)$.

- In addition to the standard Sobolev space $H^{1}(\Omega)$ we define the following spaces:

$$
H_{\Gamma_{0}}^{1}(\Omega)=\left\{w \in H^{1}(\Omega)|w|_{\Gamma_{0}}=0 \text { on } \Gamma_{0}\right\}
$$




$$
H(\operatorname{curl} ; \Omega)=\left\{w \in L^{2}\left(\Omega ; \mathbb{R}^{d}\right) \mid \operatorname{curl}(w) \in L^{2}\left(\Omega ; \mathbb{R}^{d}\right)\right\}
$$

- The third order tensor $\boldsymbol{\epsilon}_{i j k}$ is the Levi-Civita symbol that is equal to 1 if $(i, j, k)$ is an even permutation of $(1,2,3),-1$ if it is an odd permutation, and zero if any index is repeated.

- We set $\widetilde{\mathbb{L}}^{\varepsilon}(\boldsymbol{x})$ to be a general place holder under the schemes (HS 1) and (HS 2) as follows,

$$
\begin{aligned}
& \widetilde{\mathbb{L}}^{\varepsilon}(\boldsymbol{x}):=\varepsilon^{2} \mathbb{L}^{\varepsilon}(\boldsymbol{x}) \text { under the scheme (HS1), } \\
& \widetilde{\mathbb{L}}^{\varepsilon}(\boldsymbol{x}):=\mathbb{L}^{\varepsilon}(\boldsymbol{x}) \text { under the scheme (HS2). }
\end{aligned}
$$

- $\mathbb{I}_{\mathrm{D}}(\boldsymbol{x})=\left\{\begin{array}{l}1 \text { if } \boldsymbol{x} \in \mathrm{D}, \\ 0 \text { otherwise }\end{array} \quad\right.$ denotes the indicator function over some domain $\mathrm{D} \subset$ $\mathbb{R}^{d}$.

\subsection{The model}

We consider an elastic composite with periodic microstructure of period $\varepsilon$ occupying a region $\Omega \subset \mathbb{R}^{d}, d \in\{2,3\}$. The region $\Omega$ that the composite occupies, is assumed to be bounded, open, and multiply connected. $Y=(-1 / 2,1 / 2]^{d}$ is the unit cube in $\mathbb{R}^{d}$, and $\mathbb{Z}^{d}$ is the set of all $d$-dimensional vectors with integer components.

For every positive $\varepsilon$, let $N_{\varepsilon}$ be the set of all points $m \in \mathbb{Z}^{d}$ such that $\varepsilon(m+Y)$ is strictly included in $\Omega$ and denote by $\left|N_{\varepsilon}\right|$ their total number. Let $T$ be the closure of an open connected set with sufficiently smooth boundary, compactly included in $Y$. We define, for every $\varepsilon>0$ and $m \in N_{\varepsilon}, T_{m}^{\varepsilon}:=\varepsilon(m+T)$ as the region containing one of the material phases and by $S_{m}^{\varepsilon}=\partial T_{m}^{\varepsilon}$ denote the interphase boundary separating the two different material phases (see Fig. 2). We now define the following subsets of $\Omega$ :

$$
\Omega_{1 \varepsilon}:=\bigcup_{m \in N_{\varepsilon}} T_{m}^{\varepsilon}, \quad \Omega_{2 \varepsilon}:=\Omega \backslash \bar{\Omega}_{1 \varepsilon}, \quad \Omega:=\Omega_{1 \varepsilon} \cup \Omega_{2 \varepsilon} \cup\left(\cup_{m \in N_{\varepsilon}} S_{m}^{\varepsilon}\right)
$$

Moreover, we denote by $\partial \Omega$ the boundary of $\Omega$. The exterior boundary component will be denoted by $\Gamma_{0}$ and the remaining boundary components by $S_{m}^{\varepsilon}, m \in N_{\varepsilon}$. The vector $\boldsymbol{n}$ will be unit normal on $\Gamma_{0}$ pointing in the outward direction.

The heterogeneous Cosserat continuum is characterized by the following coupled system for $i \in\{1,2,3\}$,

$$
\begin{array}{cc}
-\partial_{x_{j}} \sigma_{j i}^{\varepsilon}=f_{i} & \text { in } \Omega, \\
-\partial_{x_{j}} \mu_{j i}^{\varepsilon}-\boldsymbol{\epsilon}_{i j k} \sigma_{j k}^{\varepsilon}=g_{i} & \text { in } \Omega, \\
\boldsymbol{u}^{\varepsilon}=\mathbf{0} & \text { on } \Gamma_{0}, \\
\boldsymbol{\varphi}^{\varepsilon}=\mathbf{0} & \text { on } \Gamma_{0} .
\end{array}
$$




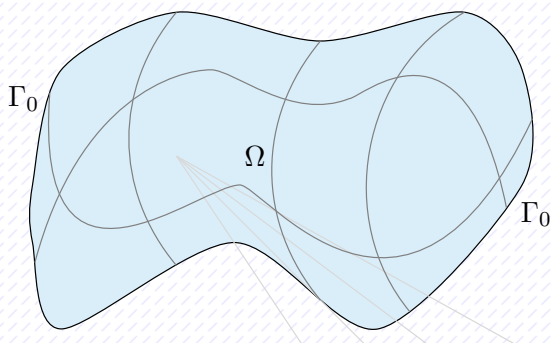

$\mathbb{R}^{d}$

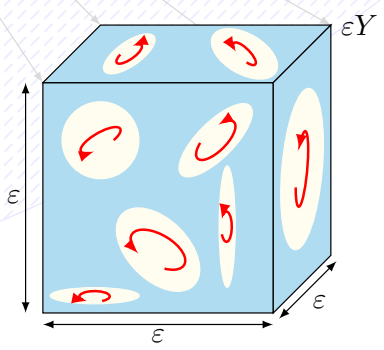

Fig. 1 Schematic of the heterogeneous Cosserat medium in vaccum

Here $\sigma^{\varepsilon}$ is the stress, $\mu^{\varepsilon}$ is the couple-stress, $\boldsymbol{u}^{\varepsilon}$ is the displacement, and $\boldsymbol{\varphi}^{\varepsilon}$ is the rotation. The system of Eq. (2.12) characterizes the mechanical deformation that the body undergoes. The equations are fully coupled and the system is closed with homogeneous Dirichlet boundary conditions on $\Gamma_{0}$. In addition to computing the displacement we must also compute the rigid rotations which makes for a fully coupled system of partial differential equations.

Constitutive relations. We content ourselves in the case of "centrosymmetric medium" where stress is related to the strain though the fourth order material tensor $\mathbb{C}^{\varepsilon}(\boldsymbol{x})$ by the relation (see [32]),

$$
\sigma^{\varepsilon}=\mathbb{C}^{\varepsilon} \gamma^{\varepsilon}
$$

while the couple-stress is related to the curvature through the fourth order material tensor $\widetilde{\mathbb{L}}^{\varepsilon}(\boldsymbol{x})$ by the relation,

$$
\mu^{\varepsilon}=\widetilde{\mathbb{L}}^{\varepsilon} \kappa^{\varepsilon}
$$


The tensors $\mathbb{C}^{\varepsilon}=\left\{C_{j i k \ell}^{\varepsilon}\right\}_{j, i, k, \ell=1}^{3}$ and $\widetilde{\mathbb{L}}^{\varepsilon}=\left\{\widetilde{L}_{j i k \ell}^{\varepsilon}\right\}_{j, i, k, \ell=1}^{3}$ are linear isotropic tensors in each phase and have the following form,

$$
\begin{aligned}
& C_{j i k \ell}^{\varepsilon}(\boldsymbol{x})=\left(\vartheta_{\varepsilon}(\boldsymbol{x})+\alpha_{\varepsilon}(\boldsymbol{x})\right) \delta_{j k} \delta_{i \ell}+\left(\vartheta_{\varepsilon}(\boldsymbol{x})-\alpha_{\varepsilon}(\boldsymbol{x})\right) \delta_{j \ell} \delta_{i k}+\varrho_{\varepsilon}(\boldsymbol{x}) \delta_{i j} \delta_{k \ell}, \\
& \widetilde{L}_{j i k \ell}^{\varepsilon}(\boldsymbol{x})=\left(\theta_{\varepsilon}(\boldsymbol{x})+\beta_{\varepsilon}(\boldsymbol{x})\right) \delta_{j k} \delta_{i \ell}+\left(\theta_{\varepsilon}(\boldsymbol{x})-\beta_{\varepsilon}(\boldsymbol{x})\right) \delta_{j \ell} \delta_{i k}+\rho_{\varepsilon}(\boldsymbol{x}) \delta_{i j} \delta_{k \ell},
\end{aligned}
$$

respectively. The coefficients $\vartheta_{\varepsilon}(\boldsymbol{x}):=\vartheta\left(\frac{x}{\varepsilon}\right), \theta_{\varepsilon}(\boldsymbol{x}):=\theta\left(\frac{\boldsymbol{x}}{\varepsilon}\right), \alpha_{\varepsilon}(\boldsymbol{x}):=\alpha\left(\frac{\boldsymbol{x}}{\varepsilon}\right)$, $\beta_{\varepsilon}(\boldsymbol{x}):=\beta\left(\frac{\boldsymbol{x}}{\varepsilon}\right), \varrho_{\varepsilon}(\boldsymbol{x}):=\varrho\left(\frac{x}{\varepsilon}\right)$, and $\rho_{\varepsilon}(\boldsymbol{x}):=\rho\left(\frac{x}{\varepsilon}\right)$, are material parameters that are piecewise constant in each phase. Thermodynamic stability relations immediately yield the symmetries, $C_{j i k \ell}^{\varepsilon}=C_{k \ell j i}^{\varepsilon}$ and $\widetilde{L}_{j i k \ell}^{\varepsilon}=\widetilde{L}_{k \ell j i}^{\varepsilon}$.

\subsection{Assumptions}

We frame the heterogeneous Cosserat continuum model $(2.12,2.13,2.14)$ under the following general assumptions:

- $\Omega$ is a bounded, multiply connected domain such that $\operatorname{mes}\left(\Gamma_{0}\right)>0, \operatorname{mes}\left(S_{\ell}^{\varepsilon}\right)>0$, and $S_{\ell}^{\varepsilon} \cap S_{p}^{\varepsilon}=\emptyset$ for $\ell, p \in N_{\varepsilon}$ with $\ell \neq p$.

- $\Gamma_{0}$ and $S_{\ell}^{\varepsilon}$ are surfaces of class $C^{2}$ and $\Gamma_{0} \cap S_{\ell}^{\varepsilon}=\emptyset$ for every $\ell \in N_{\varepsilon}$.

- The functions $\boldsymbol{f}$ and $\boldsymbol{g}$ are such that $\boldsymbol{f} \in L^{2}\left(\Omega ; \mathbb{R}^{d}\right)$ and $\boldsymbol{g} \in H(\operatorname{curl}, \Omega)$.

- The fourth order material characterization tensors are such that $C_{j i k \ell}^{\varepsilon}(\boldsymbol{x}) \in$ $\mathcal{M}_{d}^{4}(\alpha, \beta, \Omega)$ and $\widetilde{L}_{j i k \ell}^{\varepsilon}(\boldsymbol{x}) \in \mathcal{M}_{d}^{4}(\alpha, \beta, \Omega)$.

Existence and uniqueness. The Cosserat brothers [3] developed their theory to be derived from the principle of least action of Hamilton. Starting from the total energy of the system described in (2.12),

$$
\mathcal{E}_{\varepsilon}[\boldsymbol{v}, \boldsymbol{\psi}]=\frac{1}{2} \int_{\Omega} \mathbb{C}^{\varepsilon} \gamma: \gamma d \boldsymbol{x}+\frac{1}{2} \int_{\Omega} \widetilde{\mathbb{L}}^{\varepsilon} \kappa: \kappa d \boldsymbol{x}-\int_{\Omega} \boldsymbol{f} \cdot \boldsymbol{v} d \boldsymbol{x}-\int_{\Omega} \boldsymbol{g} \cdot \boldsymbol{\psi} d \boldsymbol{x},
$$

where $\mathbb{C}^{\varepsilon} \gamma: \gamma=C_{j i k \ell}^{\varepsilon} \gamma_{\ell k} \gamma_{j i}$ and $\widetilde{\mathbb{L}}^{\varepsilon} \kappa: \kappa=\widetilde{L}_{j i k \ell}^{\varepsilon} \kappa_{\ell k} \kappa_{j i}$, one can readily observe that the above energy is sequentially weakly lower semicontinuous and coersive by [20, Thm. 3.1] (see also [21,31]) in $H_{\Gamma_{0}}^{1}\left(\Omega, \mathbb{R}^{d}\right) \times H_{\Gamma_{0}}^{1}\left(\Omega, \mathbb{R}^{d}\right)$. Moreover, the following estimates hold under (HS 1) and (HS 2), respectively:

$$
\begin{aligned}
& \left(\left\|\boldsymbol{u}^{\varepsilon}\right\|_{H_{0}^{1}\left(\Omega, \mathbb{R}^{d}\right)}^{2}+\left(\left\|\boldsymbol{\varphi}^{\varepsilon}\right\|_{L^{2}\left(\Omega ; \mathbb{R}^{d}\right)}^{2}+\varepsilon\left\|\nabla \boldsymbol{\varphi}^{\varepsilon}\right\|_{L^{2}\left(\Omega ; \mathbb{R}^{d \times d}\right)}^{2}\right)\right)^{1 / 2} \\
& \quad \leq c\left(\|\boldsymbol{f}\|_{L^{2}\left(\Omega ; \mathbb{R}^{d}\right)}^{2}+\|\boldsymbol{g}\|_{L^{2}\left(\Omega ; \mathbb{R}^{d}\right)}^{2}\right)^{1 / 2}
\end{aligned}
$$




$$
\left(\left\|\boldsymbol{u}^{\varepsilon}\right\|_{H_{\Gamma_{0}}^{1}\left(\Omega ; \mathbb{R}^{d}\right)}^{2}+\left\|\boldsymbol{\varphi}^{\varepsilon}\right\|_{H_{\Gamma_{0}}^{1}\left(\Omega ; \mathbb{R}^{d}\right)}^{2}\right)^{1 / 2} \leq c\left(\|\boldsymbol{f}\|_{L^{2}\left(\Omega ; \mathbb{R}^{d}\right)}^{2}+\|\boldsymbol{g}\|_{L^{2}\left(\Omega ; \mathbb{R}^{d}\right)}^{2}\right)^{1 / 2}
$$

for some generic constant $c$ independent of $\varepsilon$. Additionally, the energy is convex in the arguments $\nabla \boldsymbol{u}$ and $\nabla \boldsymbol{\varphi}$. Hence, using the compact embedding of Rellich-Kondrachov we can apply the direct method to obtain existence and uniqueness. Furthermore, we can characterize the solution to (2.12) as the unique minimizer of,

$$
\left(\boldsymbol{u}^{\varepsilon}, \boldsymbol{\varphi}^{\varepsilon}\right)=\operatorname{argmin}_{(\boldsymbol{v}, \boldsymbol{\psi}) \in H_{\Gamma_{0}}^{1}\left(\Omega, \mathbb{R}^{d}\right) \times H_{\Gamma_{0}}^{1}\left(\Omega, \mathbb{R}^{d}\right)} \mathcal{E}_{\varepsilon}[\boldsymbol{v}, \boldsymbol{\psi}]
$$

By computing the first variation of $\mathcal{E}_{\varepsilon}$ we obtain the Euler-Lagrange equations,

$$
\int_{\Omega} \sigma_{j k}^{\varepsilon}\left(\frac{\partial v_{k}}{\partial x_{j}}-\epsilon_{i j k} \psi_{i}\right) d \boldsymbol{x}-\int_{\Omega} \mu_{j i}^{\varepsilon} \frac{\partial \psi_{i}}{\partial x_{j}} d \boldsymbol{x}-\int_{\Omega}\left(f_{i} v_{i}+g_{i} \psi_{i}\right) d \boldsymbol{x}=0 .
$$

If we group terms in the Euler-Lagrange equation above we obtain the weak form of (2.12) which reads as follows: Find $\left(\boldsymbol{u}^{\varepsilon}, \varphi^{\varepsilon}\right) \in H_{\Gamma_{0}}^{1}\left(\Omega, \mathbb{R}^{d}\right) \times H_{\Gamma_{0}}^{1}\left(\Omega, \mathbb{R}^{d}\right)$ such that,

$$
\begin{aligned}
& \int_{\Omega} \sigma_{j i}^{\varepsilon} \frac{\partial v_{i}}{\partial x_{j}} d \boldsymbol{x}-\int_{\Omega} f_{i} v_{i} d \boldsymbol{x}=0 \text { for all } \boldsymbol{v} \in H_{\Gamma_{0}}^{1}\left(\Omega, \mathbb{R}^{d}\right) \\
& \int_{\Omega} \mu_{j i}^{\varepsilon} \frac{\partial \psi_{i}}{\partial x_{j}} d \boldsymbol{x}-\int_{\Omega} \boldsymbol{\epsilon}_{i j k} \sigma_{j k}^{\varepsilon} \psi_{i} d \boldsymbol{x}-\int_{\Omega} g_{i} \psi_{i} d \boldsymbol{x}=0 \text { for all } \boldsymbol{\psi} \in H_{\Gamma_{0}}^{1}\left(\Omega, \mathbb{R}^{d}\right) .
\end{aligned}
$$

Using (2.22-2.23) we can recover the strong form of the Eq. in 2.12 in the sense of distributions as usual.

\section{Homogenization of the Cosserat continuum}

In the next two subsections we recall the definitions and properties of the periodic unfolding and averaging operators [5-8] and present our main results. We will list some of their properties, leaving the interested reader to consult $[5,6,8]$ for further details regarding proofs. 

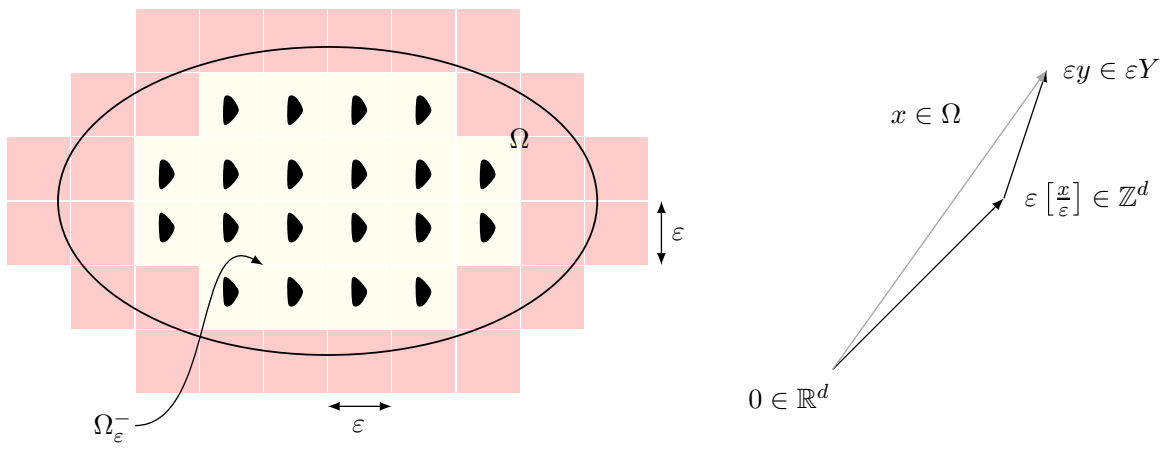

Fig. 2 Unfolding operator on a periodic grid

\subsection{The periodic unfolding and averaging operators}

We define the following domain decompositions (see [5-8]):

$$
\begin{aligned}
& K_{\varepsilon}^{-}:=\left\{\ell \in \mathbb{Z}^{d} \mid \varepsilon(\ell+Y) \subset \bar{\Omega}\right\}, \\
& \Omega_{\varepsilon}^{-}:=\operatorname{int}\left(\cup_{\ell \in K_{\varepsilon}^{-}}(\ell+Y)\right), \\
& \Lambda_{\varepsilon}^{-}:=\Omega \backslash \Omega_{\varepsilon}^{-} .
\end{aligned}
$$

Let $[z]_{Y}=\left(\left\lfloor z_{1}\right\rfloor, \ldots,\left\lfloor z_{d}\right\rfloor\right)$ denote the integer part of $z \in \mathbb{R}^{d}$ and denote by $\{z\}_{Y}$ the difference $z-[z]_{Y}$ which belongs to $Y$. Regarding our multiscale problem that depends on a small length parameter $\varepsilon>0$, we can decompose any $x \in \mathbb{R}^{d}$ using the maps $[\cdot]_{Y}: \mathbb{R}^{d} \mapsto \mathbb{Z}^{d}$ and $\{\cdot\}_{Y}: \mathbb{R}^{d} \mapsto Y$ the following way (see Fig. 1 (right)),

$$
x=\varepsilon\left(\left[\frac{x}{\varepsilon}\right]_{Y}+\left\{\frac{x}{\varepsilon}\right\}_{Y}\right) .
$$

For any Lebesgue measurable function $\varphi$ on $\Omega$ we define the periodic unfolding operator by,

$$
\mathcal{T}_{\varepsilon}(\varphi)(\boldsymbol{x}, \boldsymbol{y})= \begin{cases}\varphi\left(\varepsilon\left[\frac{\boldsymbol{x}}{\varepsilon}\right]_{Y}+\varepsilon \boldsymbol{y}\right) & \text { for a.e. }(\boldsymbol{x}, \boldsymbol{y}) \in \Omega_{\varepsilon}^{-} \times Y \\ 0 & \text { for a.e. }(\boldsymbol{x}, \boldsymbol{y}) \in \Lambda_{\varepsilon}^{-} \times Y .\end{cases}
$$

Proposition 3.1 For any $p \in[1,+\infty)$ the unfolding operator $\mathcal{T}_{\varepsilon}: L^{p}(\Omega) \mapsto L^{p}(\Omega \times$ $Y$ ) is linear, continuous, and has the following properties:

(i) $\mathcal{T}_{\mathcal{E}}(\varphi \psi)=\mathcal{T}_{\mathcal{E}}(\varphi) \mathcal{T}_{\mathcal{E}}(\psi)$ for every pair of Lebesgue measurable functions $\varphi$, $\psi$ on $\Omega$ 
(ii) For every $\varphi \in L^{1}(\Omega)$ we have,

$$
\frac{1}{|Y|} \int_{\Omega \times Y} \mathcal{T}_{\varepsilon}(\varphi)(\boldsymbol{x}, \boldsymbol{y}) d \boldsymbol{x} d \boldsymbol{y}=\int_{\Omega_{\varepsilon}^{-}} \varphi(\boldsymbol{x}) d \boldsymbol{x}=\int_{\Omega_{\varepsilon}} \varphi(\boldsymbol{x}) d \boldsymbol{x}-\int_{\Lambda_{\varepsilon}^{-}} \varphi(\boldsymbol{x}) d \boldsymbol{x}
$$

(iii) $\left\|\mathcal{T}_{\varepsilon}(\varphi)\right\|_{L^{p}(\Omega \times Y)} \leq|Y|^{1 / p}\|\varphi\|_{L^{p}(\Omega)}$ for every $\varphi \in L^{p}(\Omega)$

(iv) $\mathcal{T}_{\varepsilon}(\varphi) \rightarrow \varphi$ strongly in $L^{p}(\Omega \times Y)$ for $\varphi \in L^{p}(\Omega)$ as $\varepsilon \rightarrow 0$

(v) If $\left\{\varphi_{\varepsilon}\right\}_{\varepsilon}$ is a sequence in $L^{p}(\Omega)$ such that $\varphi_{\varepsilon} \rightarrow \varphi$ strongly in $L^{p}(\Omega)$, then $\mathcal{T}_{\varepsilon}\left(\varphi_{\varepsilon}\right) \rightarrow \varphi$ strongly in $L^{p}(\Omega \times Y)$

(vi) If $\varphi \in L^{p}(Y)$ is Y-periodic and $\varphi_{\varepsilon}(\boldsymbol{x})=\varphi\left(\frac{x}{\varepsilon}\right)$ then $\mathcal{T}_{\varepsilon}\left(\varphi_{\varepsilon}\right) \rightarrow \varphi$ strongly in $L^{p}(\Omega \times Y)$ as $\varepsilon \rightarrow 0$

(vii) If $\phi_{\varepsilon} \rightarrow \phi$ in $H^{1}(\Omega)$ then there exists an non-relabelled subsequence and a $\hat{\phi} \in$ $L^{2}\left(\Omega ; H_{\mathrm{per}}^{1}(Y)\right)$ such that

(a) $\mathcal{T}_{\varepsilon}\left(\phi_{\varepsilon}\right) \rightarrow \phi$ in $L^{2}\left(\Omega ; H^{1}(Y)\right)$

(b) $\mathcal{T}_{\varepsilon}\left(\nabla \phi_{\varepsilon}\right) \rightarrow \nabla_{x} \phi+\nabla_{y} \hat{\phi}$ in $L^{2}(\Omega \times Y)$

(viii) Let $\phi_{\varepsilon} \in H^{1}(\Omega)$ and assume that $\left\{\phi_{\varepsilon}\right\}_{\varepsilon}$ is a bounded sequence in $L^{2}(\Omega)$ satisfying $\varepsilon\left\|\nabla \phi_{\varepsilon}\right\|_{L^{2}\left(\Omega ; \mathbb{R}^{d}\right)} \leq c$ (c is a constant independent of $\varepsilon$ ) then there exists an nonrelabelled subsequence and $a \hat{\phi} \in L^{2}\left(\Omega ; H_{\mathrm{per}}^{1}(Y)\right)$ such that

(a) $\mathcal{T}_{\varepsilon}\left(\phi_{\varepsilon}\right) \rightarrow \hat{\phi}$ in $L^{2}\left(\Omega ; H^{1}(Y)\right)$

(b) $\varepsilon \mathcal{T}_{\varepsilon}\left(\nabla \phi_{\varepsilon}\right) \rightarrow \nabla_{y} \hat{\phi}$ in $L^{2}(\Omega \times Y)$

In a similar fashion we define the averaging operator $\mathfrak{U}_{\varepsilon}: L^{p}(\Omega \times Y) \rightarrow L^{p}(\Omega)$ for $p \in[1,+\infty)$, which acts as a pseudo-inverse of the unfolding operator, by:

$$
\mathcal{U}_{\varepsilon}(\Phi)(\boldsymbol{x})= \begin{cases}\int_{Y} \Phi\left(\varepsilon\left[\frac{x}{\varepsilon}\right]+\varepsilon z,\left\{\frac{x}{\varepsilon}\right\}\right) d z & \text { for a.e. } x \in \Omega_{\varepsilon}^{-} \\ 0 & \text { for a.e. } x \in \Lambda_{\varepsilon}^{-}\end{cases}
$$

Proposition 3.2 For any $p \in[1,+\infty)$ the averaging operator $\mathcal{U}_{\varepsilon}: L^{p}(\Omega \times Y) \mapsto$ $L^{p}(\Omega)$ has the following properties:

(i) If $\left\{w_{\varepsilon}\right\}_{\varepsilon} \in L^{p}(\Omega \times Y)$ is a bounded sequence such that $w_{\varepsilon} \rightarrow w$ in $L^{p}(\Omega \times Y)$ as $\varepsilon \rightarrow 0$ then

$$
\mathcal{U}_{\varepsilon}\left(w_{\varepsilon}\right) \rightarrow \int_{Y} w(\cdot, \boldsymbol{y}) d \boldsymbol{y} \text { in } L^{p}(\Omega)
$$

If $w$ is independent of $y$ then the convergence above is strong (see [6, Cor. 2.26, pg. 1599]).

(ii) If $\left\{w_{\varepsilon}\right\}_{\varepsilon}$ is a sequence in $L^{p}(\Omega)$ then the following are equivalent:

(a) $\mathcal{T}_{\varepsilon}\left(w_{\varepsilon}\right) \rightarrow \hat{w}$ in $L^{p}(\Omega \times Y)$ 
(b) $w_{\varepsilon} \mathbb{I}_{\Omega_{\varepsilon}^{-}}-\mathcal{U}_{\varepsilon}(\hat{w}) \rightarrow 0$ in $L^{p}(\Omega)$

(iii) If $\left\{w_{\varepsilon}\right\}_{\varepsilon}$ is a sequence in $L^{p}(\Omega)$ then the following are equivalent:

(a) $\mathcal{T}_{\varepsilon}\left(w_{\varepsilon}\right) \rightarrow \hat{w}$ in $L^{p}(\Omega \times Y)$ and $\int_{\Lambda_{\varepsilon}^{-}}\left|w_{\varepsilon}\right|^{p} d \boldsymbol{x} \rightarrow 0$

(b) $w_{\varepsilon}-\mathcal{U}_{\varepsilon}(\hat{w}) \rightarrow 0$ in $L^{p}(\Omega)$

\subsection{Main results}

\subsubsection{Homogenization under the HS 1 scheme}

Theorem 3.1 If $\left(\boldsymbol{u}^{\varepsilon}, \varphi^{\varepsilon}\right)$ is the solution set to (2.12) then, under the HS 1 scheme, there exist $\boldsymbol{u}^{0} \in H_{\Gamma_{0}}^{1}\left(\Omega ; \mathbb{R}^{d}\right), \boldsymbol{u}^{1} \in L^{2}\left(\Omega ; H_{\text {per }}^{1}\left(Y ; \mathbb{R}^{d}\right)\right), \boldsymbol{\varphi}^{0} \in L^{2}\left(\Omega ; H_{\mathrm{per}}^{1}\left(Y ; \mathbb{R}^{d}\right)\right)$ such that,

$$
\begin{gathered}
\mathcal{T}_{\varepsilon}\left(\boldsymbol{u}^{\varepsilon}\right) \rightarrow \boldsymbol{u}^{0} \text { in } L^{2}\left(\Omega ; H^{1}\left(Y ; \mathbb{R}^{d}\right)\right), \\
\mathcal{T}_{\mathcal{E}}\left(\nabla \boldsymbol{u}^{\varepsilon}\right) \rightarrow \nabla_{x} \boldsymbol{u}^{0}+\nabla_{y} \boldsymbol{u}^{1} \text { in } L^{2}\left(\Omega \times Y ; \mathbb{R}^{d \times d}\right), \\
\mathcal{T}_{\varepsilon}\left(\boldsymbol{\varphi}^{\varepsilon}\right) \rightarrow \boldsymbol{\varphi}^{0} \text { in } L^{2}\left(\Omega ; H^{1}\left(Y ; \mathbb{R}^{d}\right)\right),
\end{gathered}
$$

and $\left(\boldsymbol{u}^{0}, \boldsymbol{\varphi}^{0}, \boldsymbol{u}^{1}\right)$ is the unique solution set of,

$$
\begin{aligned}
& \int_{\Omega \times Y} C_{j i k \ell}(\boldsymbol{y})\left(\partial_{x_{\ell}} u_{k}^{0}+\partial_{x_{\ell}} u_{k}^{1}-\boldsymbol{\epsilon}_{v k \ell} \varphi_{v}^{0}-\frac{1}{2} \boldsymbol{\epsilon}_{v i j} g_{v}\right)\left(\partial_{x_{i}} V_{j}+\partial_{y_{i}} \bar{W}_{j}\right) d \boldsymbol{y} d \boldsymbol{x} \\
& -\frac{1}{2} \int_{\Omega \times Y} \boldsymbol{\epsilon}_{v i j} g_{v}\left(\partial_{x_{i}} V_{j}+\partial_{y_{i}} \bar{W}_{j}\right) d \boldsymbol{y} d \boldsymbol{x}+\int_{\Omega} f_{i} V_{i} d \boldsymbol{x}=0,
\end{aligned}
$$

for all $\boldsymbol{V} \in H_{0}^{1}\left(\Omega ; \mathbb{R}^{d}\right)$ and $\overline{\boldsymbol{W}} \in L^{2}\left(\Omega ; H^{1}\left(Y ; \mathbb{R}^{d}\right)\right)$. Furthermore, (3.10) is equivalent to the following homogenized linear elasticity problem,

$$
\int_{\Omega} \sigma^{\mathrm{eff}}: \nabla_{x} \boldsymbol{V} d \boldsymbol{x}=\frac{1}{2} \int_{\Omega} \boldsymbol{g} \cdot \operatorname{curl}_{x} \boldsymbol{V} d \boldsymbol{x}+\int_{\Omega} \boldsymbol{f} \cdot \boldsymbol{V} d \boldsymbol{x},
$$

if $\boldsymbol{u}^{1}$ and $\boldsymbol{\varphi}^{0}$ have the following form,

$$
\begin{aligned}
& u_{i}^{1}(\boldsymbol{x}, \boldsymbol{y})=\zeta_{i}^{k \ell}(\boldsymbol{y}) \partial_{x_{\ell}} u_{k}^{0}(\boldsymbol{x})+c_{i}(\boldsymbol{x}), \\
& \varphi_{\nu}^{0}(\boldsymbol{x}, \boldsymbol{y})=\xi_{v}^{k \ell}(\boldsymbol{y}) \partial_{x_{\ell}} u_{k}^{0}(\boldsymbol{x}),
\end{aligned}
$$

and we select $\overline{\boldsymbol{W}} \equiv \mathbf{0}$ in (3.10). Here $\sigma_{i j}^{\mathrm{eff}}:=\left(C_{i j p q}^{\mathrm{eff}} \partial_{x_{q}} u_{p}^{0}-\frac{1}{2} \boldsymbol{\epsilon}_{v i j} g_{v}\right)$ is the Cauchy stress,

$$
C_{j i p q}^{\mathrm{eff}}=\int_{Y} C_{j i k \ell}(\boldsymbol{y})\left(\delta_{k p} \delta_{q \ell}+\partial_{y_{\ell}} \zeta_{k}^{p q}-\boldsymbol{\epsilon}_{v k \ell} \xi_{\nu}^{p q}\right) d \boldsymbol{y}
$$


are the effective coefficients, and $\zeta^{k \ell}$ and $\xi^{k \ell}$ are the local solutions satisfying the variational problems,

$$
\begin{gathered}
\int_{Y} C_{j i k \ell}(\boldsymbol{y})\left(\frac{1}{2} \delta_{k p} \delta_{q \ell}+\partial_{y_{\ell}} \zeta_{k}^{p q}\right) \partial_{y_{i}} v_{j} d \boldsymbol{y}=0 \text { for all } \boldsymbol{v} \in H_{\mathrm{per}}^{1}\left(Y ; \mathbb{R}^{d}\right), \\
\int_{Y} C_{j i k \ell}(\boldsymbol{y})\left(\frac{1}{2} \delta_{k p} \delta_{q \ell}+\boldsymbol{\epsilon}_{v k \ell} \xi_{v}^{p q}\right) \partial_{y_{i}} v_{j} d \boldsymbol{y}=0 \text { for all } \boldsymbol{v} \in H_{\mathrm{per}}^{1}\left(Y ; \mathbb{R}^{d}\right) .
\end{gathered}
$$

Proof Using (2.18) and Proposition 3.1 vii. and viii. we obtain (3.7-3.9). Additionally, the weak form (2.21) of the Cosserat continuum reads: Find $\left(\boldsymbol{u}^{\varepsilon}, \boldsymbol{\varphi}^{\varepsilon}\right) \in$ $H_{\Gamma_{0}}^{1}\left(\Omega_{0} ; \mathbb{R}^{d}\right) \times H_{\Gamma_{0}}^{1}\left(\Omega ; \mathbb{R}^{d}\right)$ such that,

$$
\begin{aligned}
& \int_{\Omega} C_{j i k \ell}\left(\frac{\boldsymbol{x}}{\varepsilon}\right)\left(\partial_{x_{\ell}} u_{k}^{\varepsilon}-\boldsymbol{\epsilon}_{v k \ell} \varphi_{\nu}^{\varepsilon}\right)\left(\partial_{x_{j}} v_{i}-\boldsymbol{\epsilon}_{v i j} \psi_{v}\right) d \boldsymbol{x} \\
& -\int_{\Omega} \varepsilon^{2} L_{j i k \ell}\left(\frac{\boldsymbol{x}}{\varepsilon}\right) \partial_{x_{\ell}} \varphi_{k}^{\varepsilon} \partial_{x_{i}} \psi_{j} d \boldsymbol{x}-\int_{\Omega}\left(f_{i} v_{i}+g_{i} \psi_{i}\right) d \boldsymbol{x}=0 .
\end{aligned}
$$

Unfold the above expression using Proposition 3.1 properties $i ., i i .$, and $i v$ ) and obtain,

$$
\begin{aligned}
& \int_{\Omega \times Y} C_{j i k \ell}(\boldsymbol{y})\left(\mathcal{T}_{\varepsilon}\left(\partial_{x_{\ell}} u_{k}^{\varepsilon}\right)-\boldsymbol{\epsilon}_{v k \ell} \mathcal{T}_{\varepsilon}\left(\varphi_{\nu}^{\varepsilon}\right)\right)\left(\mathcal{T}_{\varepsilon}\left(\partial_{x_{j}} v_{i}\right)-\boldsymbol{\epsilon}_{v i j} \mathcal{T}_{\varepsilon}\left(\psi_{v}\right)\right) d \boldsymbol{y} d \boldsymbol{x} \\
& -\int_{\Omega \times Y} \varepsilon^{2} L_{j i k \ell}(\boldsymbol{y}) \mathcal{I}_{\varepsilon}\left(\partial_{x_{\ell}} \varphi_{k}^{\varepsilon}\right) \mathcal{T}_{\varepsilon}\left(\partial_{x_{i}} \psi_{j}\right) d \boldsymbol{y} d \boldsymbol{x} \\
& -\int_{\Omega \times Y}\left(\mathcal{T}_{\varepsilon}\left(f_{i}\right) \mathcal{T}_{\varepsilon}\left(v_{i}\right)+\mathcal{T}_{\varepsilon}\left(g_{i}\right) \mathcal{T}_{\varepsilon}\left(\psi_{i}\right)\right) d \boldsymbol{y} d \boldsymbol{x}=0 .
\end{aligned}
$$

Set $\boldsymbol{v}:=\boldsymbol{V}(\boldsymbol{x})$ and $\boldsymbol{\psi}:=\boldsymbol{\Psi}(\boldsymbol{x})$ for any test functions $\boldsymbol{V} \in C_{0}^{\infty}\left(\Omega ; \mathbb{R}^{d}\right)$ and $\Psi \in C_{0}^{\infty}\left(\Omega ; \mathbb{R}^{d}\right)$ in (3.18) and let $\varepsilon \rightarrow 0$ to obtain,

$$
\begin{aligned}
& \int_{\Omega \times Y} C_{j i k \ell}(\boldsymbol{y})\left(\partial_{x_{\ell}} u_{k}^{0}+\partial_{y_{\ell}} u_{k}^{1}-\boldsymbol{\epsilon}_{v k \ell} \varphi_{\nu}^{0}(\boldsymbol{x}, \boldsymbol{y})\right)\left(\partial_{x_{j}} V_{i}-\boldsymbol{\epsilon}_{v i j} \Psi_{v}\right) d \boldsymbol{y} d \boldsymbol{x} \\
& \quad-\int_{\Omega}\left(f_{i} V_{i}+g_{i} \Psi_{i}\right) d \boldsymbol{x}=0
\end{aligned}
$$

Select now test functions of the form $\boldsymbol{v}=\boldsymbol{v}^{\varepsilon}:=\varepsilon U(\boldsymbol{x}) \boldsymbol{W}\left(\frac{\boldsymbol{x}}{\varepsilon}\right)$ where $U \in C_{0}^{\infty}(\Omega)$ and $\boldsymbol{W} \in H_{\mathrm{per}}^{1}\left(Y ; \mathbb{R}^{d}\right)$. It is cleat that $\boldsymbol{v}^{\varepsilon} \rightarrow \mathbf{0}$ in $L^{2}\left(\Omega ; \mathbb{R}^{d}\right)$. Moreover, we have $\partial_{x_{j}} v_{i}^{\varepsilon}(\boldsymbol{x})=\varepsilon \partial_{x_{j}} U(\boldsymbol{x}) W_{i}(\boldsymbol{x} / \varepsilon)+U(\boldsymbol{x}) \partial_{y_{j}} W_{i}(\boldsymbol{x} / \varepsilon)$ which implies $\mathcal{T}_{\varepsilon}\left(\partial_{x_{j}} v_{i}^{\varepsilon}\right) \rightarrow$ $\partial_{y_{j}} \bar{W}_{i}(\boldsymbol{x}, \boldsymbol{y})$ in $L^{2}(\Omega \times Y)$ as $\varepsilon \rightarrow 0$ where $\bar{W}_{i}(\boldsymbol{x}, \boldsymbol{y}):=U(\boldsymbol{x}) W_{i}(\boldsymbol{y})$. Likewise, we select as test function for the rotations $\boldsymbol{\psi}=\boldsymbol{\psi}^{\varepsilon}:=\varepsilon \Phi(\boldsymbol{x}) \boldsymbol{\Xi}\left(\frac{\boldsymbol{x}}{\varepsilon}\right)$ where $\Phi \in C_{0}^{\infty}(\Omega)$ and $\boldsymbol{\Xi} \in H_{\mathrm{per}}^{1}\left(Y ; \mathbb{R}^{d}\right)$ with $\boldsymbol{\psi}^{\varepsilon} \rightarrow \mathbf{0}$ in $L^{2}\left(\Omega ; \mathbb{R}^{d}\right)$ and $\mathcal{T}_{\varepsilon}\left(\partial_{x_{j}} \psi_{i}^{\varepsilon}\right) \rightarrow \partial_{y_{j}} \bar{\Xi}_{i}(\boldsymbol{x}, \boldsymbol{y})$ in $L^{2}(\Omega \times Y)$ as $\varepsilon \rightarrow 0$ where $\bar{\Xi}_{i}(\boldsymbol{x}, \boldsymbol{y}):=\Psi(\boldsymbol{x}) \boldsymbol{\Xi}_{i}(\boldsymbol{y})$. Hence, unfolding (3.18) with 
the above test functions we obtain,

$$
\begin{aligned}
& \int_{\Omega \times Y} C_{j i k \ell}(\boldsymbol{y})\left(\mathcal{T}_{\varepsilon}\left(\partial_{x_{\ell}} u_{k}^{\varepsilon}\right)-\boldsymbol{\epsilon}_{v k \ell} \mathcal{T}_{\varepsilon}\left(\varphi_{\nu}^{\varepsilon}\right)\right)\left(\mathcal{T}_{\varepsilon}\left(\partial_{x_{j}} v_{i}^{\varepsilon}\right)-\boldsymbol{\epsilon}_{v i j} \mathcal{T}_{\varepsilon}\left(\psi_{\nu}^{\varepsilon}\right)\right) d \boldsymbol{y} d \boldsymbol{x} \\
& -\int_{\Omega \times Y} \varepsilon^{2} L_{j i k \ell}(\boldsymbol{y}) \mathcal{T}_{\varepsilon}\left(\partial_{x_{\ell}} \varphi_{k}^{\varepsilon}\right) \mathcal{T}_{\varepsilon}\left(\partial_{x_{i}} \psi_{j}^{\varepsilon}\right) d \boldsymbol{y} d \boldsymbol{x} \\
& -\int_{\Omega \times Y}\left(\mathcal{T}_{\varepsilon}\left(f_{i}\right) \mathcal{T}_{\varepsilon}\left(v_{i}^{\varepsilon}\right)+\mathcal{T}_{\varepsilon}\left(g_{i}\right) \mathcal{T}_{\varepsilon}\left(\psi_{i}^{\varepsilon}\right)\right) d \boldsymbol{y} d \boldsymbol{x}=0 .
\end{aligned}
$$

Letting $\varepsilon$ tend to zero in (3.20) we obtain,

$$
\int_{\Omega \times Y} C_{j i k \ell}(\boldsymbol{y})\left(\partial_{x_{\ell}} u_{k}^{0}+\partial_{y_{\ell}} u_{k}^{1}-\boldsymbol{\epsilon}_{v k \ell} \varphi_{v}^{0}(\boldsymbol{x}, \boldsymbol{y})\right) U(\boldsymbol{x}) \partial_{y_{j}} W_{i}(\boldsymbol{y}) d \boldsymbol{y} d \boldsymbol{x}=0
$$

Defining $\overline{\boldsymbol{W}}(\boldsymbol{x}, \boldsymbol{y}):=U(\boldsymbol{x}) \boldsymbol{W}(\boldsymbol{y})$ and adding (3.19) and (3.21) we obtain,

$$
\begin{aligned}
& \int_{\Omega \times Y}\left\{C_{j i k \ell}(\boldsymbol{y})\left(\partial_{x_{\ell}} u_{k}^{0}+\partial_{y_{\ell}} u_{k}^{1}-\boldsymbol{\epsilon}_{v k \ell} \varphi_{\nu}^{0}(\boldsymbol{x}, \boldsymbol{y})\right)\right\}\left(\partial_{x_{j}} V_{i}+\partial_{y_{j}} \bar{W}_{i}-\boldsymbol{\epsilon}_{v i j} \Psi_{v}\right) d \boldsymbol{y} d \boldsymbol{x} \\
& \left.-\int_{\Omega}\left\{f_{i} V_{i}+g_{i} \Psi_{i}\right)\right\} d \boldsymbol{y} d \boldsymbol{x}=0 .
\end{aligned}
$$

By the density of $C_{0}^{\infty}(\Omega) \otimes H_{\text {per }}^{1}\left(Y ; \mathbb{R}^{d}\right)$ in $L^{2}\left(\Omega ; H_{\text {per }}^{1}\left(Y ; \mathbb{R}^{d}\right)\right)$ the result holds for all $\overline{\boldsymbol{W}}(\boldsymbol{x}, \boldsymbol{y}) \in L^{2}\left(\Omega ; H_{\mathrm{per}}^{1}\left(Y ; \mathbb{R}^{d}\right)\right)$.

The above equation is not immediately in a form that we recognize. However, using the properties of the Levi-Civita tensor, we can re-write $g_{i}=\frac{1}{2} \epsilon_{i p q} \boldsymbol{\epsilon}_{j p q} g_{j}$. Grouping the terms in (3.22) by test function we obtain,

$$
\begin{aligned}
& \int_{\Omega \times Y}\left\{C_{j i k \ell}(\boldsymbol{y})\left(\partial_{x_{\ell}} u_{k}^{0}+\partial_{y_{\ell}} u_{k}^{1}-\boldsymbol{\epsilon}_{v k \ell} \varphi_{\nu}^{0}(\boldsymbol{x}, \boldsymbol{y})\right)+\frac{1}{2} \boldsymbol{\epsilon}_{s i j} g_{s}\right\}\left(\partial_{x_{j}} V_{i}+\partial_{y_{j}} \bar{W}_{i}\right) d \boldsymbol{y} d \boldsymbol{x} \\
& \left.-\int_{\Omega \times Y}\left\{C_{j i k \ell}(\boldsymbol{y})\left(\partial_{x_{\ell}} u_{k}^{0}+\partial_{y_{\ell}} u_{k}^{1}-\boldsymbol{\epsilon}_{v k \ell} \varphi_{\nu}^{0}(\boldsymbol{x}, \boldsymbol{y})\right)+\frac{1}{2} \boldsymbol{\epsilon}_{s i j} g_{s}\right\} \boldsymbol{\epsilon}_{v i j} \Psi_{v}\right) d \boldsymbol{y} d \boldsymbol{x} \\
& -\int_{\Omega}\left\{f_{i} V_{i}+\frac{1}{2} \boldsymbol{\epsilon}_{s i j} g_{s} \partial_{x_{j}} V_{i}\right\} d \boldsymbol{y} d \boldsymbol{x}=0 .
\end{aligned}
$$

From here we can obtain two sets of equations. By considering, first, that $(\boldsymbol{V}, \overline{\boldsymbol{W}}, \boldsymbol{\Psi})=(\boldsymbol{V}, \overline{\boldsymbol{W}}, \mathbf{0})$ we have,

$$
\begin{gathered}
\int_{\Omega \times Y}\left\{C_{j i k \ell}(\boldsymbol{y})\left(\partial_{x_{\ell}} u_{k}^{0}+\partial_{y_{\ell}} u_{k}^{1}-\boldsymbol{\epsilon}_{\nu k \ell} \varphi_{\nu}^{0}(\boldsymbol{x}, \boldsymbol{y})\right)+\frac{1}{2} \boldsymbol{\epsilon}_{s i j} g_{s}\right\}\left(\partial_{x_{j}} V_{i}+\partial_{y_{j}} \bar{W}_{i}\right) d \boldsymbol{y} d \boldsymbol{x} \\
-\int_{\Omega}\left\{f_{i} V_{i}+\frac{1}{2} \boldsymbol{\epsilon}_{s i j} g_{s}\left(\partial_{x_{j}} V_{i}+\partial_{y_{j}} \bar{W}_{i}\right)\right\} d \boldsymbol{y} d \boldsymbol{x}=0 .
\end{gathered}
$$


By considering $(\boldsymbol{V}, \overline{\boldsymbol{W}}, \boldsymbol{\Psi})=(\mathbf{0}, \mathbf{0}, \boldsymbol{\Psi})$ we have,

$$
-\int_{\Omega \times Y}\left\{C_{j i k \ell}(\boldsymbol{y})\left(\partial_{x_{\ell}} u_{k}^{0}+\partial_{y_{\ell}} u_{k}^{1}-\boldsymbol{\epsilon}_{v k \ell} \varphi_{\nu}^{0}(\boldsymbol{x}, \boldsymbol{y})\right)+\frac{1}{2} \boldsymbol{\epsilon}_{s i j} g_{s}\right\} \boldsymbol{\epsilon}_{v i j} \Psi_{v} d \boldsymbol{y} d \boldsymbol{x}=0
$$

If in (3.24) we select $\boldsymbol{V} \equiv \mathbf{0}$ we can see that both $\boldsymbol{u}^{1}$ and $\varphi^{0}$ depend on $\nabla_{x} \boldsymbol{u}^{0}$ linearly. In some sense, this could be interpreted as the macroscopic displacement having contributions from the microscopic displacement and rotation, independently. Hence, the form of $\boldsymbol{u}^{1}$ and $\boldsymbol{\varphi}^{0}$ look as follows,

$$
\begin{aligned}
& u_{i}^{1}(\boldsymbol{x}, \boldsymbol{y})=\zeta_{i}^{k \ell}(\boldsymbol{y}) \partial_{x_{\ell}} u_{k}^{0}(\boldsymbol{x})+c_{i}(\boldsymbol{x}) \\
& \varphi_{v}^{0}(\boldsymbol{x}, \boldsymbol{y})=\xi_{v}^{k \ell}(\boldsymbol{y}) \partial_{x_{\ell}} u_{k}^{0}(\boldsymbol{x}),
\end{aligned}
$$

where the correctors $\zeta^{k \ell}$ and $\boldsymbol{\xi}^{k \ell}$ are the local solutions satisfying the following variational problems,

$$
\begin{aligned}
& \boldsymbol{\zeta}^{p q} \in H_{\mathrm{per}}^{1}\left(Y ; \mathbb{R}^{d}\right), \quad \int_{Y} \zeta^{p q} d \boldsymbol{y}=0 \\
& \int_{Y} C_{j i k \ell}(\boldsymbol{y})\left(\frac{1}{2} \delta_{k p} \delta_{q \ell}+\partial_{y_{\ell}} \zeta_{k}^{p q}\right) \partial_{y_{i}} v_{j} d \boldsymbol{y}=0 \text { for all } \boldsymbol{v} \in H_{\mathrm{per}}^{1}\left(Y ; \mathbb{R}^{d}\right) \\
& \boldsymbol{\xi}^{p q} \in H_{\mathrm{per}}^{1}\left(Y ; \mathbb{R}^{d}\right), \quad \int_{Y} \boldsymbol{\xi}^{p q} d \boldsymbol{y}=0 \\
& \int_{Y} C_{j i k \ell}(\boldsymbol{y})\left(\frac{1}{2} \delta_{k p} \delta_{q \ell}+\boldsymbol{\epsilon}_{v k \ell} \xi_{v}^{p q}\right) \partial_{y_{i}} v_{j} d \boldsymbol{y}=0 \text { for all } \boldsymbol{v} \in H_{\mathrm{per}}^{1}\left(Y ; \mathbb{R}^{d}\right)
\end{aligned}
$$

Existence and uniqueness for (3.28) follows from classical theory of variational inequalities [25]. While for problem (3.29) one can show existence (up to an additive constant) as in [17, Thm. 3.4, pg. 45].

Returning to (3.24) and substituting $\overline{\boldsymbol{W}}=\mathbf{0}, \boldsymbol{u}^{1}$ and $\boldsymbol{\varphi}^{0}$ from (3.26 and 3.27) respectively, we obtain,

$$
\int_{\Omega} \sigma_{i j}^{\mathrm{eff}} \partial_{x_{j}} V_{i} d \boldsymbol{x}=\frac{1}{2} \int_{\Omega} \boldsymbol{\epsilon}_{v i j} g_{v} \partial_{x_{i}} V_{j} d \boldsymbol{x}+\int_{\Omega} f_{i} V_{i} d \boldsymbol{x}
$$

where

$$
\sigma_{i j}^{\mathrm{eff}}:=\left(C_{j i p q}^{\mathrm{eff}} \partial_{x_{q}} u_{p}^{0}-\frac{1}{2} \epsilon_{v i j} g_{\nu}\right),
$$

and

$$
C_{j i p q}^{\mathrm{eff}}=\int_{Y} C_{j i k \ell}(\boldsymbol{y})\left(\delta_{k p} \delta_{q \ell}+\partial_{y_{\ell}} \zeta_{k}^{p q}-\boldsymbol{\epsilon}_{\nu \ell \ell} \xi_{v}^{p q}\right) d \boldsymbol{y} .
$$


Using Eq. (3.25) we see that $\sigma_{i j}^{\text {eff }}=\sigma_{j i}^{\text {eff }}$ exactly like the Cauchy stress in classical linear elasticity. These are precisely the homogenized equations obtained by [13] using two-scale expansions under their scheme HS1.

Remark 3.1 Equation (3.29) is an additional local problem that does not appear in the homogenization of classical linear elasticity. Its appearance is solely a contribution of the non-symmetric part of the strain tensor. Upon closer examination, local problem (3.29) is one that involves rotations (or curls) which implies that certain curvature-twist effects are present in the microscale. Moreover, these curvature-twist effects manifest themselves macroscopically as part of the effective material tensor of a linear elastic material and not independently.

Proposition 3.3 If we use the notation $\gamma_{j i}^{0}:=\partial_{x_{j}} u_{i}^{0}$, and $\gamma_{j i}^{1}:=\partial_{y_{j}} u_{i}^{1}-\epsilon_{k j i} \varphi_{k}^{0}$ then under the assumptions of Theorem 3.1 we have the following convergence results,

$$
\begin{aligned}
& \lim _{\varepsilon \rightarrow 0} \int_{\Omega} \mathbb{C}^{\varepsilon}(\boldsymbol{x}) \gamma^{\varepsilon}: \gamma^{\varepsilon} d \boldsymbol{x}=\int_{\Omega \times Y} \mathbb{C}(\boldsymbol{y})\left(\gamma^{0}+\gamma^{1}\right):\left(\gamma^{0}+\gamma^{1}\right) d \boldsymbol{y} d \boldsymbol{x} \\
& \lim _{\varepsilon \rightarrow 0} \int_{\Lambda_{\varepsilon}^{-}} \mathbb{C}^{\varepsilon}(\boldsymbol{x}) \gamma^{\varepsilon}: \gamma^{\varepsilon} d \boldsymbol{x}=0 .
\end{aligned}
$$

Proof Using the weak lower semicontinuity of the integrals, the fact that tensors $\mathbb{C}^{\varepsilon}$ and $\widetilde{\mathbb{L}}^{\varepsilon}$ belong in $\mathcal{M}_{d}^{4}(\alpha, \beta, \Omega)$, and properties of the limit infimum we obtain,

$$
\begin{aligned}
& \int_{\Omega \times Y} \mathbb{C}(\boldsymbol{y})\left(\gamma^{0}+\gamma^{1}\right):\left(\gamma^{0}+\gamma^{1}\right) d \boldsymbol{y} d \boldsymbol{x} \\
& \leq \liminf _{\varepsilon \rightarrow 0} \int_{\Omega \times Y} \mathcal{T}_{\varepsilon}\left(\mathbb{C}^{\varepsilon}\right) \mathcal{T}_{\varepsilon}\left(\gamma^{\varepsilon}\right): \mathcal{T}_{\varepsilon}\left(\gamma^{\varepsilon}\right) d \boldsymbol{y} d \boldsymbol{x} \\
& \leq \liminf _{\varepsilon \rightarrow 0} \int_{\Omega \times Y} \mathcal{T}_{\varepsilon}\left(\mathbb{C}^{\varepsilon}\right) \mathcal{T}_{\varepsilon}\left(\gamma^{\varepsilon}\right): \mathcal{T}_{\varepsilon}\left(\gamma^{\varepsilon}\right) d \boldsymbol{y} d \boldsymbol{x} \\
& +\liminf _{\varepsilon \rightarrow 0} \int_{\Omega \times Y} \mathcal{T}_{\varepsilon}\left(\tilde{\mathbb{L}}^{\varepsilon}\right) \mathcal{T}_{\varepsilon}\left(\kappa^{\varepsilon}\right): \mathcal{T}_{\varepsilon}\left(\kappa^{\varepsilon}\right) d \boldsymbol{y} d \boldsymbol{x} \\
& \leq \liminf _{\varepsilon \rightarrow 0}\left\{\int_{\Omega \times Y} \mathcal{T}_{\varepsilon}\left(\mathbb{C}^{\varepsilon}\right) \mathcal{T}_{\varepsilon}\left(\gamma^{\varepsilon}\right): \mathcal{T}_{\varepsilon}\left(\gamma^{\varepsilon}\right) d \boldsymbol{y} d \boldsymbol{x}\right. \\
& \left.+\int_{\Omega \times Y} \mathcal{T}_{\varepsilon}\left(\tilde{\mathbb{L}}^{\varepsilon}\right) \mathcal{T}_{\varepsilon}\left(\kappa^{\varepsilon}\right): \mathcal{T}_{\varepsilon}\left(\kappa^{\varepsilon}\right) d \boldsymbol{y} d \boldsymbol{x}\right\} \\
& \leq \liminf _{\varepsilon \rightarrow 0}\left\{\int_{\Omega} \mathbb{C}^{\varepsilon} \gamma^{\varepsilon}: \gamma^{\varepsilon} d \boldsymbol{x}+\int_{\Omega} \widetilde{\mathbb{L}}^{\varepsilon} \kappa^{\varepsilon}: \kappa^{\varepsilon} d \boldsymbol{x}\right\} \\
& =\liminf _{\varepsilon \rightarrow 0} \int_{\Omega \times Y}\left(\boldsymbol{f} \cdot \boldsymbol{u}^{\varepsilon}+\boldsymbol{g} \cdot \boldsymbol{\varphi}^{\varepsilon}\right) d \boldsymbol{y} d \boldsymbol{x} \\
& =\int_{\Omega \times Y}\left(\boldsymbol{f} \cdot \boldsymbol{u}^{0}+\boldsymbol{g} \cdot \boldsymbol{\varphi}^{0}\right) d \boldsymbol{y} d \boldsymbol{x} \\
& =\int_{\Omega \times Y} \mathbb{C}(\boldsymbol{y})\left(\gamma^{0}+\gamma^{1}\right):\left(\gamma^{0}+\gamma^{1}\right) d \boldsymbol{y} d \boldsymbol{x},
\end{aligned}
$$


which is precisely (3.33). We remark that the last equality came from equation (3.22). Moreover, (3.33) implies (3.34).

Remark 3.2 Immediately one can observe from Proposition 3.3 that the following result holds,

$$
\lim _{\varepsilon \rightarrow 0} \int_{\Omega \times Y} \mathcal{T}_{\varepsilon}\left(\mathbb{C}^{\varepsilon}\right) \mathcal{T}_{\varepsilon}\left(\gamma^{\varepsilon}\right): \mathcal{T}_{\varepsilon}\left(\gamma^{\varepsilon}\right) d \boldsymbol{y} d \boldsymbol{x}=\int_{\Omega \times Y} \mathbb{C}(\boldsymbol{y})\left(\gamma^{0}+\gamma^{1}\right):\left(\gamma^{0}+\gamma^{1}\right) d \boldsymbol{y} d \boldsymbol{x}
$$

Corollary 3.1 The Cosserat strain $\gamma^{\varepsilon}$ converges strongly in $L^{2}\left(\Omega \times Y ; \mathbb{R}^{d \times d}\right)$,

$$
\lim _{\varepsilon \rightarrow 0} \mathcal{T}_{\mathcal{E}}\left(\gamma^{\varepsilon}\right) \rightarrow \gamma^{0}+\gamma^{1} \text { in } L^{2}\left(\Omega \times Y ; \mathbb{R}^{d \times d}\right)
$$

Proof By expanding the square of the expression below we have,

$$
\begin{aligned}
& \int_{\Omega \times Y} \mathbb{C}(\boldsymbol{y})\left(\mathcal{T}_{\varepsilon}\left(\gamma^{\varepsilon}\right)-\gamma^{0}-\gamma^{1}\right):\left(\mathcal{T}_{\varepsilon}\left(\gamma^{\varepsilon}\right)-\gamma^{0}-\gamma^{1}\right) d \boldsymbol{y} d \boldsymbol{x} \\
& =\int_{\Omega \times Y} \mathbb{C}(\boldsymbol{y}) \mathcal{T}_{\varepsilon}\left(\gamma^{\varepsilon}\right): \mathcal{T}_{\varepsilon}\left(\gamma^{\varepsilon}\right) d \boldsymbol{y} d \boldsymbol{x}-\int_{\Omega \times Y} \mathbb{C}(\boldsymbol{y}) \mathcal{T}_{\varepsilon}\left(\gamma^{\varepsilon}\right):\left(\gamma^{0}-\gamma^{1}\right) d \boldsymbol{y} d \boldsymbol{x} \\
& \quad-\int_{\Omega \times Y} \mathbb{C}(\boldsymbol{y})\left(\gamma^{0}-\gamma^{1}\right): \mathcal{T}_{\varepsilon}\left(\gamma^{\varepsilon}\right) d \boldsymbol{y} d \boldsymbol{x} \\
& \quad+\int_{\Omega \times Y} \mathbb{C}(\boldsymbol{y})\left(\gamma^{0}-\gamma^{1}\right):\left(\gamma^{0}-\gamma^{1}\right) d \boldsymbol{y} d \boldsymbol{x} .
\end{aligned}
$$

The first term converges from (3.36) while the rest of the terms converge by (3.7) and properties of the unfolding operator. Hence, all the terms on the right hand side above sum to zero in the limit and the result follows.

\subsubsection{Homogenization under the HS 2 scheme}

Theorem 3.2 If $\left(\boldsymbol{u}^{\varepsilon}, \varphi^{\varepsilon}\right)$ is the solution set to (2.12) then, under the HS 2 scheme, there exist $\boldsymbol{u}^{0} \in H_{\Gamma_{0}}^{1}\left(\Omega ; \mathbb{R}^{d}\right), \boldsymbol{u}^{1} \in L^{2}\left(\Omega ; H_{p e r}^{1}\left(Y ; \mathbb{R}^{d}\right)\right), \varphi^{0} \in H_{\Gamma_{0}}^{1}\left(\Omega ; \mathbb{R}^{d}\right), \varphi^{1} \in$ $L^{2}\left(\Omega ; H_{p e r}^{1}\left(Y ; \mathbb{R}^{d}\right)\right)$ such that,

$$
\begin{gathered}
\mathcal{T}_{\varepsilon}\left(\boldsymbol{u}^{\varepsilon}\right) \rightarrow \boldsymbol{u}^{0} \text { in } L^{2}\left(\Omega ; H^{1}\left(Y ; \mathbb{R}^{d}\right)\right), \\
\mathcal{T}_{\varepsilon}\left(\nabla \boldsymbol{u}^{\varepsilon}\right) \rightarrow \nabla_{x} \boldsymbol{u}^{0}+\nabla_{y} \boldsymbol{u}^{1} \text { in } L^{2}\left(\Omega \times Y ; \mathbb{R}^{d \times d}\right), \\
\mathcal{T}_{\varepsilon}\left(\boldsymbol{\varphi}^{\varepsilon}\right) \rightarrow \boldsymbol{\varphi}^{0} \text { in } L^{2}\left(\Omega ; H^{1}\left(Y ; \mathbb{R}^{d}\right)\right), \\
\mathcal{T}_{\varepsilon}\left(\nabla \boldsymbol{\varphi}^{\varepsilon}\right) \rightarrow \nabla_{x} \boldsymbol{\varphi}^{0}+\nabla_{y} \boldsymbol{\varphi}^{1} \text { in } L^{2}\left(\Omega \times Y ; \mathbb{R}^{d \times d}\right),
\end{gathered}
$$


and $\left(\boldsymbol{u}^{0}, \boldsymbol{u}^{1}, \varphi^{0}, \varphi^{1}\right)$ is the unique solution set of

$$
\begin{aligned}
& \int_{\Omega \times Y} C_{j i k \ell}(\boldsymbol{y})\left(\partial_{x_{\ell}} u_{k}^{0}+\partial_{x_{\ell}} u_{k}^{1}-\boldsymbol{\epsilon}_{v k \ell} \varphi_{\nu}^{0}\right)\left(\partial_{x_{i}} V_{j}+\partial_{y_{i}} \bar{W}_{j}-\boldsymbol{\epsilon}_{v i j} \Psi_{\nu}\right) d \boldsymbol{y} d \boldsymbol{x} \\
& -\int_{\Omega \times Y} L_{j i k \ell}(\boldsymbol{y})\left(\partial_{x_{\ell}} \varphi_{k}^{0}+\partial_{y_{\ell}} \varphi_{k}^{1}\right)\left(\partial_{x_{i}} \Psi_{j}+\partial_{y_{i}} \bar{\Xi}_{j}\right) d \boldsymbol{y} d \boldsymbol{x} \\
& \quad-\int_{\Omega} f_{i} V_{i}+g_{i} \Psi_{i} d \boldsymbol{x}=0
\end{aligned}
$$

for all $\boldsymbol{V} \in H_{0}^{1}\left(\Omega ; \mathbb{R}^{d}\right)$ and $\overline{\boldsymbol{W}} \in L^{2}\left(\Omega ; H^{1}\left(Y ; \mathbb{R}^{d}\right)\right)$. Furthermore, (3.43) is equivalent to,

$$
\begin{aligned}
& \int_{\Omega} C_{j i p q}^{\mathrm{eff}}\left(\partial_{x_{p}} u_{q}^{0}-\boldsymbol{\epsilon}_{v p q} \varphi_{\nu}^{0}\right)\left(\partial_{x_{i}} V_{j}-\boldsymbol{\epsilon}_{v i j} \Psi_{v}\right) d \boldsymbol{x}-\int_{\Omega} L_{j i p q}^{\mathrm{eff}} \partial_{x_{p}} \varphi_{q}^{0} \partial_{x_{i}} \Psi_{j} d \boldsymbol{x} \\
& \quad-\int_{\Omega}\left(f_{i} V_{i}+g_{i} \Psi_{i}\right) d \boldsymbol{x}=0
\end{aligned}
$$

if $\boldsymbol{u}^{1}$ and $\boldsymbol{\varphi}^{1}$ have the form,

$$
\begin{aligned}
& u_{i}^{1}(\boldsymbol{x}, \boldsymbol{y})=\zeta_{i}^{p q}(\boldsymbol{y})\left(\partial_{x_{p}} u_{q}^{0}(\boldsymbol{x})-\boldsymbol{\epsilon}_{v p q} \varphi_{v}^{0}(\boldsymbol{x})\right)+\kappa_{i}(\boldsymbol{x}), \\
& \varphi_{v}^{1}(\boldsymbol{x}, \boldsymbol{y})=\xi_{v}^{p q}(\boldsymbol{y}) \partial_{x_{p}} \varphi_{q}^{0}(\boldsymbol{x})+\kappa_{i}(\boldsymbol{x})
\end{aligned}
$$

and we set $\overline{\boldsymbol{W}} \equiv \mathbf{0}$ and $\overline{\boldsymbol{\Xi}} \equiv \mathbf{0}$. Here,

$$
\begin{aligned}
C_{j i p q}^{\mathrm{eff}} & =\int_{Y} C_{j i k \ell}(\boldsymbol{y})\left(\delta_{k p} \delta_{\ell q}+\partial_{y_{\ell}} \zeta_{k}^{p q}\right) d \boldsymbol{y} \\
L_{j i p q}^{\mathrm{eff}} & =\int_{Y} L_{j i k \ell}(\boldsymbol{y})\left(\delta_{k p} \delta_{\ell q}+\partial_{y_{\ell}} \xi_{k}^{p q}\right) d \boldsymbol{y}
\end{aligned}
$$

are the effective Cosserat coeffcients with $\boldsymbol{\zeta}^{p q}$ and $\boldsymbol{\xi}^{p q}$ being the local solutions on the unit cell satisfying the second order elliptic problems,

$$
\begin{aligned}
& \boldsymbol{\zeta}^{p q} \in H_{\mathrm{per}}^{1}\left(Y ; \mathbb{R}^{d}\right), \quad \int_{Y} \boldsymbol{\zeta}^{p q} d \boldsymbol{y}=0 \\
& \int_{Y} C_{j i k \ell}(\boldsymbol{y})\left(\delta_{k p} \delta_{q \ell}+\partial_{y_{\ell}} \zeta_{k}^{p q}\right) \partial_{y_{i}} w_{j} d \boldsymbol{y}=0 \text { for all } \boldsymbol{w} \in H_{\mathrm{per}}^{1}\left(Y ; \mathbb{R}^{d}\right)(3 \\
& \boldsymbol{\xi}^{p q} \in H_{\mathrm{per}}^{1}\left(Y ; \mathbb{R}^{d}\right), \quad \int_{Y} \boldsymbol{\xi}^{p q} d \boldsymbol{y}=0 \\
& \quad \int_{Y} L_{j i k \ell}(\boldsymbol{y})\left(\delta_{k p} \delta_{q \ell}+\partial_{y_{\ell}} \xi_{k}^{p q}\right) \partial_{y_{i}} v_{j} d \boldsymbol{y}=0 \text { for all } \boldsymbol{v} \in H_{\mathrm{per}}^{1}\left(Y ; \mathbb{R}^{d}\right)
\end{aligned}
$$


Proof Once again, using (2.19) and Proposition 3.1 vii. we obtain (3.39-3.42) as $\varepsilon \rightarrow 0$. Furthermore, the weak form (2.21) of the Cosserat continuum reads: Find $\left(\boldsymbol{u}^{\varepsilon}, \boldsymbol{\varphi}^{\varepsilon}\right) \in H_{\Gamma_{0}}^{1}\left(\Omega ; \mathbb{R}^{d}\right) \times H_{\Gamma_{0}}^{1}\left(\Omega ; \mathbb{R}^{d}\right)$ such that,

$$
\begin{aligned}
\int_{\Omega} C_{j i k \ell}\left(\frac{\boldsymbol{x}}{\varepsilon}\right) & \left(\partial_{x_{\ell}} u_{k}^{\varepsilon}-\boldsymbol{\epsilon}_{v k \ell} \varphi_{\nu}^{\varepsilon}\right)\left(\partial_{x_{j}} v_{i}-\boldsymbol{\epsilon}_{v i j} \psi_{\nu}\right) d \boldsymbol{x} \\
& -\int_{\Omega} L_{j i k \ell}\left(\frac{\boldsymbol{x}}{\varepsilon}\right) \partial_{x_{\ell}} \varphi_{k}^{\varepsilon} \partial_{x_{i}} \psi_{j} d \boldsymbol{x}-\int_{\Omega}\left(f_{i} v_{i}+g_{i} \psi_{i}\right) d \boldsymbol{x}=0 .
\end{aligned}
$$

Unfold the above expression using Proposition 3.1 properties $i ., i i .$, and $i v$ ) and obtain,

$$
\begin{aligned}
& \int_{\Omega \times Y} C_{j i k \ell}(\boldsymbol{y})\left(\mathcal{T}_{\varepsilon}\left(\partial_{x_{\ell}} u_{k}^{\varepsilon}\right)-\boldsymbol{\epsilon}_{v k \ell} \mathcal{T}_{\varepsilon}\left(\varphi_{\nu}^{\varepsilon}\right)\right)\left(\mathcal{T}_{\varepsilon}\left(\partial_{x_{j}} v_{i}\right)-\boldsymbol{\epsilon}_{v i j} \mathcal{T}_{\varepsilon}\left(\psi_{v}\right)\right) d \boldsymbol{y} d \boldsymbol{x} \\
& -\int_{\Omega \times Y} L_{j i k \ell}(\boldsymbol{y}) \mathcal{T}_{\varepsilon}\left(\partial_{x_{\ell}} \varphi_{k}^{\varepsilon}\right) \mathcal{T}_{\varepsilon}\left(\partial_{x_{i}} \psi_{j}\right) d \boldsymbol{y} d \boldsymbol{x} \\
& -\int_{\Omega \times Y}\left(\mathcal{T}_{\varepsilon}\left(f_{i}\right) \mathcal{T}_{\varepsilon}\left(v_{i}\right)+\mathcal{T}_{\varepsilon}\left(g_{i}\right) \mathcal{T}_{\varepsilon}\left(\psi_{i}\right)\right) d \boldsymbol{y} d \boldsymbol{x}=0
\end{aligned}
$$

Select the same test functions as before. Namely, set $\boldsymbol{v}:=\boldsymbol{V}(\boldsymbol{x})$ and $\boldsymbol{\psi}:=\boldsymbol{\Psi}(\boldsymbol{x})$ for any test functions $\boldsymbol{V} \in C_{0}^{\infty}\left(\Omega ; \mathbb{R}^{d}\right)$ and $\Psi \in C_{0}^{\infty}\left(\Omega ; \mathbb{R}^{d}\right)$ in (3.52) and let $\varepsilon \rightarrow 0$ to obtain,

$$
\begin{aligned}
& \int_{\Omega \times Y} C_{j i k \ell}(\boldsymbol{y})\left(\partial_{x_{\ell}} u_{k}^{0}+\partial_{x_{\ell}} u_{k}^{1}-\boldsymbol{\epsilon}_{v k \ell} \varphi_{\nu}^{0}\right)\left(\partial_{x_{i}} V_{j}-\boldsymbol{\epsilon}_{v i j} \Psi_{v}\right) d \boldsymbol{y} d \boldsymbol{x} \\
& -\int_{\Omega \times Y} L_{j i k \ell}(\boldsymbol{y})\left(\partial_{x_{\ell}} \varphi_{k}^{0}+\partial_{y_{\ell}} \varphi_{k}^{1}\right) \partial_{x_{i}} \Psi_{j} d \boldsymbol{y} d \boldsymbol{x} \\
& -\int_{\Omega}\left(f_{i} V_{i}+g_{i} \Psi_{i}\right) d \boldsymbol{x}=0,
\end{aligned}
$$

Select now the test functions from before $\boldsymbol{v}=\boldsymbol{v}^{\varepsilon}:=\varepsilon U(\boldsymbol{x}) \boldsymbol{W}\left(\frac{\boldsymbol{x}}{\varepsilon}\right)$ and $\boldsymbol{\psi}=$ $\boldsymbol{\psi}^{\varepsilon}:=\varepsilon \Phi(\boldsymbol{x}) \boldsymbol{\Xi}\left(\frac{\boldsymbol{x}}{\varepsilon}\right)$. Recall that $\boldsymbol{v}^{\varepsilon} \rightarrow 0, \boldsymbol{\psi}^{\varepsilon} \rightarrow \mathbf{0}$ in $L^{2}\left(\Omega ; \mathbb{R}^{d}\right)$ and $\mathcal{T}_{\varepsilon}\left(\partial_{x_{j}} \psi_{i}^{\varepsilon}\right) \rightarrow$ $\partial_{y_{j}} \overline{\boldsymbol{\Xi}}_{i}(\boldsymbol{x}, \boldsymbol{y}), \mathcal{T}_{\varepsilon}\left(\partial_{x_{j}} v_{i}^{\varepsilon}\right) \rightarrow \partial_{y_{j}} \bar{W}_{i}(\boldsymbol{x}, \boldsymbol{y})$ in $L^{2}(\Omega \times Y)$.

With the above test functions we obtain,

$$
\begin{gathered}
\int_{\Omega \times Y} C_{j i k \ell}(\boldsymbol{y})\left(\partial_{x_{\ell}} u_{k}^{0}+\partial_{x_{\ell}} u_{k}^{1}-\boldsymbol{\epsilon}_{\nu k \ell} \varphi_{\nu}^{0}\right) \partial_{y_{i}} \bar{W}_{j} d \boldsymbol{y} d \boldsymbol{x} \\
-\int_{\Omega \times Y} L_{j i k \ell}(\boldsymbol{y})\left(\partial_{x_{\ell}} \varphi_{k}^{0}+\partial_{y_{\ell}} \varphi_{k}^{1}\right) \partial_{y_{i}} \overline{\boldsymbol{\Xi}}_{j} d \boldsymbol{y} d \boldsymbol{x}=0
\end{gathered}
$$

Adding (3.53 and 3.54) we obtain,

$$
\begin{gathered}
\int_{\Omega \times Y} C_{j i k \ell}(\boldsymbol{y})\left(\partial_{x_{\ell}} u_{k}^{0}+\partial_{x_{\ell}} u_{k}^{1}-\boldsymbol{\epsilon}_{v k \ell} \varphi_{\nu}^{0}\right)\left(\partial_{x_{j}} V_{i}+\partial_{y_{i}} \bar{W}_{j}-\boldsymbol{\epsilon}_{v i j} \Psi_{v}\right) d \boldsymbol{y} d \boldsymbol{x} \\
-\int_{\Omega \times Y} L_{j i k \ell}(\boldsymbol{y})\left(\partial_{x_{\ell}} \varphi_{k}^{0}+\partial_{y_{\ell}} \varphi_{k}^{1}\right)\left(\partial_{x_{j}} \Psi_{i}+\partial_{y_{i}} \bar{\Xi}_{j}\right) d \boldsymbol{y} d \boldsymbol{x}
\end{gathered}
$$




$$
-\int_{\Omega}\left(f_{i} V_{i}+g_{i} \Psi_{i}\right) d \boldsymbol{x}=0
$$

Using the density of $C_{0}^{\infty}\left(\Omega ; \mathbb{R}^{d}\right)$ in $H_{\Gamma_{0}}^{1}\left(\Omega ; \mathbb{R}^{d}\right)$ and $C_{0}^{\infty}(\Omega) \otimes H_{\mathrm{per}}^{1}\left(Y ; \mathbb{R}^{d}\right)$ in $L^{2}\left(\Omega ; H_{\text {per }}^{1}\left(Y ; \mathbb{R}^{d}\right)\right)$ the result holds true for all $\boldsymbol{V} \in H_{\Gamma_{0}}^{1}\left(\Omega ; \mathbb{R}^{d}\right)$ and all $\overline{\boldsymbol{W}} \in$ $L^{2}\left(\Omega ; H_{\text {per }}^{1}\left(Y ; \mathbb{R}^{d}\right)\right)$.

Taking $(\boldsymbol{V}, \overline{\boldsymbol{W}}, \boldsymbol{\Psi}, \overline{\boldsymbol{\Xi}})=(\boldsymbol{V}, \overline{\boldsymbol{W}}, \mathbf{0}, \mathbf{0})$ we obtain,

$$
\int_{\Omega \times Y} C_{j i k \ell}(\boldsymbol{y})\left(\partial_{x_{\ell}} u_{k}^{0}+\partial_{x_{\ell}} u_{k}^{1}-\boldsymbol{\epsilon}_{\nu k \ell} \varphi_{\nu}^{0}\right)\left(\partial_{x_{j}} V_{i}+\partial_{y_{i}} \bar{W}_{j}\right) d \boldsymbol{y} d \boldsymbol{x}-\int_{\Omega} f_{i} V_{i} d \boldsymbol{x}=0 .
$$

Taking $(\boldsymbol{V}, \overline{\boldsymbol{W}}, \boldsymbol{\Psi}, \overline{\boldsymbol{\Xi}})=(\mathbf{0}, \mathbf{0}, \boldsymbol{\Psi}, \overline{\boldsymbol{\Xi}})$ we obtain,

$$
\begin{aligned}
& -\int_{\Omega \times Y} C_{j i k \ell}(\boldsymbol{y})\left(\partial_{x_{\ell}} u_{k}^{0}+\partial_{x_{\ell}} u_{k}^{1}-\boldsymbol{\epsilon}_{v k \ell} \varphi_{\nu}^{0}\right) \boldsymbol{\epsilon}_{v i j} \Psi_{\nu} d \boldsymbol{y} d \boldsymbol{x} \\
& -\int_{\Omega \times Y} L_{j i k \ell}(\boldsymbol{y})\left(\partial_{x_{\ell}} \varphi_{k}^{0}+\partial_{y_{\ell}} \varphi_{k}^{1}\right)\left(\partial_{x_{j}} \Psi_{i}+\partial_{y_{i}} \overline{\boldsymbol{\Xi}}_{j}\right) d \boldsymbol{y} d \boldsymbol{x} \\
& \quad-\int_{\Omega} g_{i} \Psi_{i} d \boldsymbol{x}=0 .
\end{aligned}
$$

Moreover, if we select $\boldsymbol{V} \equiv \mathbf{0}$ in (3.56) we obtain that $\boldsymbol{u}^{1}$ has the following form,

$$
u_{i}^{1}(\boldsymbol{x}, \boldsymbol{y})=\zeta_{i}^{p q}(\boldsymbol{y})\left(\partial_{x_{p}} u_{q}^{0}(\boldsymbol{x})-\boldsymbol{\epsilon}_{v p q} \varphi_{\nu}^{0}(\boldsymbol{x})\right)+\kappa_{i}(\boldsymbol{x}) .
$$

While, if we set $\boldsymbol{\Psi} \equiv \mathbf{0}$ in (3.57) we obtain $\varphi^{1}$ the following form,

$$
\varphi_{\nu}^{1}(\boldsymbol{x}, \boldsymbol{y})=\xi_{\nu}^{p q}(\boldsymbol{y}) \partial_{x_{p}} \varphi_{q}^{0}(\boldsymbol{x})+\kappa_{i}(\boldsymbol{x})
$$

where $\boldsymbol{\zeta}^{p q}$ and $\boldsymbol{\xi}^{p q}$ satisfy (3.49) and (3.50), respectively and $\kappa_{i}(\boldsymbol{x})$ is some generic constant function in $\boldsymbol{y}$. We remark that existence and uniqueness for $\boldsymbol{\zeta}^{p q}$ and $\boldsymbol{\xi}^{p q}$ follow from the theory of variational inequalities.

Rewriting, (3.55) and substituting in $\overline{\boldsymbol{W}} \equiv \mathbf{0}, \overline{\boldsymbol{\Xi}} \equiv \mathbf{0}, \boldsymbol{u}^{1}$, and $\boldsymbol{\varphi}^{1}$ from (3.56) and (3.57), respectively and factoring out common terms we obtain,

$$
\begin{aligned}
& \int_{\Omega} C_{j i p q}^{\mathrm{eff}}\left(\partial_{x_{p}} u_{q}^{0}-\boldsymbol{\epsilon}_{v p q} \varphi_{\nu}^{0}\right)\left(\partial_{x_{i}} V_{j}-\boldsymbol{\epsilon}_{v i j} \Psi_{v}\right) d \boldsymbol{x}-\int_{\Omega} L_{j i p q}^{\mathrm{eff}} \partial_{x_{p}} \varphi_{q}^{0} \partial_{x_{i}} \Psi_{j} d \boldsymbol{x} \\
& \quad-\int_{\Omega}\left(f_{i} V_{i}+g_{i} \Psi_{i}\right) d \boldsymbol{x}=0
\end{aligned}
$$

where

$$
C_{j i p q}^{\mathrm{eff}}=\int_{Y} C_{j i k \ell}(\boldsymbol{y})\left(\delta_{k p} \delta_{\ell q}+\partial_{y_{\ell}} \zeta_{k}^{p q}\right) d \boldsymbol{y}
$$




$$
L_{j i p q}^{\mathrm{eff}}=\int_{Y} L_{j i k \ell}(\boldsymbol{y})\left(\delta_{k p} \delta_{\ell q}+\partial_{y_{\ell}} \xi_{k}^{p q}\right) d \boldsymbol{y}
$$

Remark 3.3 Under the HS 2 scheme we obtain an effective Cosserat continuum in (3.60). As a result we have two sets of effective coefficients in (3.61) and (3.62). One that relates the non-symmetric strain tensor to the stress and one that relates the curvature-twist tensor to the couple stress. Unlike in the first homogenization scheme, HS 1, the curvature-twist effects manifest themselves as a separate equation.

Proposition 3.4 If we use the notation, $\gamma_{j i}^{0}:=\partial_{x_{j}} u_{i}^{0}-\epsilon_{k j i} \varphi_{k}^{0}, \gamma_{j i}^{1}:=\partial_{y_{j}} u_{i}^{1}-\epsilon_{k j i} \varphi_{k}^{1}$, $\kappa^{0}:=\partial_{x_{j}} \varphi_{i}^{0}$, and $\kappa^{1}:=\partial_{y_{j}} \varphi_{i}^{1}$ then under the assumptions of Theorem 3.2 we have the following convergence results,

$$
\begin{aligned}
& \lim _{\varepsilon \rightarrow 0} \int_{\Omega}\left(\mathbb{C}^{\varepsilon}(\boldsymbol{x}) \gamma^{\varepsilon}: \gamma^{\varepsilon}+\widetilde{\mathbb{L}}^{\varepsilon}(\boldsymbol{x}) \kappa^{\varepsilon}: \kappa^{\varepsilon}\right) d \boldsymbol{x} \\
& =\int_{\Omega \times Y}\left(\mathbb{C}(\boldsymbol{y})\left(\gamma^{0}+\gamma^{1}\right):\left(\gamma^{0}+\gamma^{1}\right)+\mathbb{L}(\boldsymbol{y})\left(\kappa^{0}+\kappa^{1}\right):\left(\kappa^{0}+\kappa^{1}\right)\right) d \boldsymbol{y} d \boldsymbol{x} \\
& \lim _{\varepsilon \rightarrow 0} \int_{\Lambda_{\varepsilon}^{-}}\left(\mathbb{C}^{\varepsilon}(\boldsymbol{x}) \gamma^{\varepsilon}: \gamma^{\varepsilon}+\widetilde{\mathbb{L}}^{\varepsilon}(\boldsymbol{x}) \kappa^{\varepsilon}: \kappa^{\varepsilon}\right) d \boldsymbol{x}=0 .
\end{aligned}
$$

Proof As before, using the weak lower semicontinuity of the integrals, the fact that tensors $\mathbb{C}^{\varepsilon}$ and $\tilde{\mathbb{L}}^{\varepsilon}$ belong in $\mathcal{M}_{d}^{4}(\alpha, \beta, \Omega)$, and properties of the limit infimum we obtain,

$$
\begin{array}{rl}
\int_{\Omega \times Y} & \mathbb{C}(\boldsymbol{y})\left(\gamma^{0}+\gamma^{1}\right):\left(\gamma^{0}+\gamma^{1}\right) d \boldsymbol{y} d \boldsymbol{x}+\int_{\Omega \times Y} \mathbb{L}(\boldsymbol{y})\left(\kappa^{0}+\kappa^{1}\right):\left(\kappa^{0}+\kappa^{1}\right) d \boldsymbol{y} d \boldsymbol{x} \\
\leq & \liminf _{\varepsilon \rightarrow 0} \int_{\Omega \times Y} \mathcal{T}_{\varepsilon}\left(\mathbb{C}^{\varepsilon}\right) \mathcal{T}_{\varepsilon}\left(\gamma^{\varepsilon}\right): \mathcal{T}_{\varepsilon}\left(\gamma^{\varepsilon}\right) d \boldsymbol{y} d \boldsymbol{x} \\
& +\liminf _{\varepsilon \rightarrow 0} \int_{\Omega \times Y} \mathcal{T}_{\varepsilon}\left(\widetilde{\mathbb{L}}^{\varepsilon}\right) \mathcal{T}_{\varepsilon}\left(\kappa^{\varepsilon}\right): \mathcal{T}_{\varepsilon}\left(\kappa^{\varepsilon}\right) d \boldsymbol{y} d \boldsymbol{x} \\
\leq & \liminf _{\varepsilon \rightarrow 0}\left\{\int_{\Omega \times Y} \mathcal{T}_{\varepsilon}\left(\mathbb{C}^{\varepsilon}\right) \mathcal{T}_{\varepsilon}\left(\gamma^{\varepsilon}\right): \mathcal{T}_{\varepsilon}\left(\gamma^{\varepsilon}\right) d \boldsymbol{y} d \boldsymbol{x}\right. \\
& \left.+\int_{\Omega \times Y} \mathcal{T}_{\varepsilon}\left(\widetilde{\mathbb{L}}^{\varepsilon}\right) \mathcal{T}_{\varepsilon}\left(\kappa^{\varepsilon}\right): \mathcal{T}_{\varepsilon}\left(\kappa^{\varepsilon}\right) d \boldsymbol{y} d \boldsymbol{x}\right\} \\
\leq & \liminf _{\varepsilon \rightarrow 0}\left\{\int_{\Omega} \mathbb{C}^{\varepsilon} \gamma^{\varepsilon}: \gamma^{\varepsilon} d \boldsymbol{x}+\int_{\Omega} \tilde{\mathbb{L}}^{\varepsilon} \kappa^{\varepsilon}: \kappa^{\varepsilon} d \boldsymbol{x}\right\} \\
= & \liminf _{\varepsilon \rightarrow 0} \int_{\Omega \times Y}\left(\boldsymbol{f} \cdot \boldsymbol{u}^{\varepsilon}+\boldsymbol{g} \cdot \boldsymbol{\varphi}^{\varepsilon}\right) d \boldsymbol{y} d \boldsymbol{x} \\
= & \int_{\Omega \times Y}\left(\boldsymbol{f} \cdot \boldsymbol{u}^{0}+\boldsymbol{g} \cdot \boldsymbol{\varphi}^{0}\right) d \boldsymbol{y} d \boldsymbol{x} \\
= & \int_{\Omega \times Y} \mathbb{C}(\boldsymbol{y})\left(\gamma^{0}+\gamma^{1}\right):\left(\gamma^{0}+\gamma^{1}\right) d \boldsymbol{y} d \boldsymbol{x}+\int_{\Omega \times Y} \mathbb{L}(\boldsymbol{y})
\end{array}
$$




$$
\left(\kappa^{0}+\kappa^{1}\right):\left(\kappa^{0}+\kappa^{1}\right) d \boldsymbol{y} d \boldsymbol{x},
$$

which is precisely (3.63). We remark that the last equality came from equation (3.55). Moreover, (3.63) implies (3.64).

Remark 3.4 Immediately one can observe from Proposition 3.4 that the following result holds,

$$
\begin{aligned}
& \lim _{\varepsilon \rightarrow 0} \int_{\Omega \times Y}\left(\mathcal{T}_{\varepsilon}\left(\mathbb{C}^{\varepsilon}\right) \mathcal{T}_{\varepsilon}\left(\gamma^{\varepsilon}\right): \mathcal{T}_{\varepsilon}\left(\gamma^{\varepsilon}\right)+\mathcal{T}_{\mathcal{\varepsilon}}\left(\widetilde{\mathbb{L}}^{\varepsilon}\right) \mathcal{T}_{\varepsilon}\left(\kappa^{\varepsilon}\right): \mathcal{T}_{\varepsilon}\left(\kappa^{\varepsilon}\right)\right) d \boldsymbol{y} d \boldsymbol{x} \\
& \quad=\int_{\Omega \times Y} \mathbb{C}(\boldsymbol{y})\left(\gamma^{0}+\gamma^{1}\right):\left(\gamma^{0}+\gamma^{1}\right) d \boldsymbol{y} d \boldsymbol{x}+\int_{\Omega \times Y} \mathbb{L}(\boldsymbol{y})\left(\kappa^{0}+\kappa^{1}\right):\left(\kappa^{0}+\kappa^{1}\right) d \boldsymbol{y} d \boldsymbol{x}
\end{aligned}
$$

Corollary 3.2 The following convergence results hold for the Cosserat strain and curvature-twist tensors,

$$
\begin{aligned}
& \lim _{\varepsilon \rightarrow 0} \mathcal{T}_{\varepsilon}\left(\gamma^{\varepsilon}\right) \rightarrow \gamma^{0}+\gamma^{1} \text { in } L^{2}\left(\Omega \times Y ; \mathbb{R}^{d \times d}\right) \\
& \lim _{\varepsilon \rightarrow 0} \mathcal{T}_{\mathcal{E}}\left(\kappa^{\varepsilon}\right) \rightarrow \kappa^{0}+\kappa^{1} \text { in } L^{2}\left(\Omega \times Y ; \mathbb{R}^{d \times d}\right)
\end{aligned}
$$

Proof By expanding the square of the expressions below we have,

$$
\begin{aligned}
& \int_{\Omega \times Y} \mathbb{C}(\boldsymbol{y})\left(\mathcal{T}_{\varepsilon}\left(\gamma^{\varepsilon}\right)-\gamma^{0}-\gamma^{1}\right):\left(\mathcal{T}_{\varepsilon}\left(\gamma^{\varepsilon}\right)-\gamma^{0}-\gamma^{1}\right) d \boldsymbol{y} d \boldsymbol{x} \\
& \quad+\int_{\Omega \times Y} \mathbb{L}(\boldsymbol{y})\left(\mathcal{T}_{\varepsilon}\left(\kappa^{\varepsilon}\right)-\kappa^{0}-\kappa^{1}\right):\left(\mathcal{T}_{\varepsilon}\left(\kappa^{\varepsilon}\right)-\kappa^{0}-\kappa^{1}\right) d \boldsymbol{y} d \boldsymbol{x} \\
& =\int_{\Omega \times Y} \mathbb{C}(\boldsymbol{y}) \mathcal{T}_{\varepsilon}\left(\gamma^{\varepsilon}\right): \mathcal{T}_{\varepsilon}\left(\gamma^{\varepsilon}\right) d \boldsymbol{y} d \boldsymbol{x}-\int_{\Omega \times Y} \mathbb{C}(\boldsymbol{y}) \mathcal{T}_{\varepsilon}\left(\gamma^{\varepsilon}\right):\left(\gamma^{0}-\gamma^{1}\right) d \boldsymbol{y} d \boldsymbol{x} \\
& \quad-\int_{\Omega \times Y} \mathbb{C}(\boldsymbol{y})\left(\gamma^{0}-\gamma^{1}\right): \mathcal{T}_{\varepsilon}\left(\gamma^{\varepsilon}\right) d \boldsymbol{y} d \boldsymbol{x}+\int_{\Omega \times Y} \mathbb{C}(\boldsymbol{y})\left(\gamma^{0}-\gamma^{1}\right):\left(\gamma^{0}-\gamma^{1}\right) d \boldsymbol{y} d \boldsymbol{x} \\
& \quad+\int_{\Omega \times Y} \mathbb{L}(\boldsymbol{y}) \mathcal{T}_{\varepsilon}\left(\kappa^{\varepsilon}\right): \mathcal{T}_{\varepsilon}\left(\kappa^{\varepsilon}\right) d \boldsymbol{y} d \boldsymbol{x}-\int_{\Omega \times Y} \mathbb{L}(\boldsymbol{y}) \mathcal{T}_{\mathcal{\varepsilon}}\left(\kappa^{\varepsilon}\right):\left(\kappa^{0}-\kappa^{1}\right) d \boldsymbol{y} d \boldsymbol{x} \\
& \quad-\int_{\Omega \times Y} \mathbb{L}(\boldsymbol{y})\left(\kappa^{0}-\kappa^{1}\right): \mathcal{T}_{\varepsilon}\left(\kappa^{\varepsilon}\right) d \boldsymbol{y} d \boldsymbol{x}+\int_{\Omega \times Y} \mathbb{L}(\boldsymbol{y})\left(\kappa^{0}-\kappa^{1}\right):\left(\kappa^{0}-\kappa^{1}\right) d \boldsymbol{y} d \boldsymbol{x}
\end{aligned}
$$

Combining terms, using (3.36) and (3.39-3.42) we obtain that the right hand side is zero and the results follow.

\section{Some results regarding correctors}

In this section, we provide some results that can be interpreted as "corrector" type results for a Cosserat continuum under each homogenization scheme. Classical correctors in the theory of homogenization for linear elasticity, transform the weak converge 
of the displacement $\boldsymbol{u}^{\varepsilon}-\boldsymbol{u}^{0}$ to strong convergence by subtracting a term involving the gradient of $\boldsymbol{u}^{0}$ and the local solutions on the unit cell. Given that kinematics of a Cosserat continuum are more convoluted, it is not immediately clear what the form of the corrector should be.

The correctors are obtained using the averaging operator in (3.5) as is done in [6]. Hence, we do not require any additional regularity assumptions of the local solutions $(3.28,3.29,3.49),(3.50)$ as is done in standard homogenization problems (see e.g. [33]).

\subsection{Correctors under HS 1 scheme}

Theorem 4.1 Under the assumptions of Theorem 3.1 we have the following strong convergence,

$$
\gamma^{\varepsilon}-\gamma^{0}-\mathcal{U}_{\varepsilon}\left(\gamma^{1}\right) \rightarrow 0 \text { in } L^{2}\left(\Omega, \mathbb{R}^{d \times d}\right)
$$

Proof Using $(3.33,3.34,3.37)$, and Proposition 3.2 iii. we have,

$$
\gamma^{\varepsilon}-\mathcal{U}_{\varepsilon}\left(\gamma^{0}\right)-\mathcal{U}_{\varepsilon}\left(\gamma^{1}\right) \rightarrow 0 \text { in } L^{2}\left(\Omega ; \mathbb{R}^{d \times d}\right) .
$$

Since $\gamma^{0}$ is independent of $y$ we can use Proposition $3.2 i$ to obtain,

$$
\mathcal{U}_{\varepsilon}\left(\gamma^{0}\right) \rightarrow \gamma^{0} \text { in } L^{2}\left(\Omega ; \mathbb{R}^{d \times d}\right)
$$

Hence, the result follows.

\subsection{Correctors under HS 2 scheme}

Theorem 4.2 Under the assumptions of Theorem 3.2 we have the following strong convergence results,

$$
\begin{gathered}
\gamma^{\varepsilon}-\gamma^{0}-\mathcal{U}_{\varepsilon}\left(\gamma^{1}\right) \rightarrow 0 \text { in } L^{2}\left(\Omega, \mathbb{R}^{d \times d}\right) \\
\kappa^{\varepsilon}-\kappa^{0}-\mathcal{U}_{\varepsilon}\left(\kappa^{1}\right) \rightarrow 0 \text { in } L^{2}\left(\Omega, \mathbb{R}^{d \times d}\right)
\end{gathered}
$$

Proof Using $(3.63,3.64,3.67,3.68)$ and Proposition 3.2 iii. we have,

$$
\begin{gathered}
\gamma^{\varepsilon}-\mathcal{U}_{\varepsilon}\left(\gamma^{0}\right)-\mathcal{U}_{\varepsilon}\left(\gamma^{1}\right) \rightarrow 0 \text { in } L^{2}\left(\Omega ; \mathbb{R}^{d \times d}\right) . \\
\kappa^{\varepsilon}-\mathcal{U}_{\varepsilon}\left(\kappa^{0}\right)-\mathcal{U}_{\varepsilon}\left(\kappa^{1}\right) \rightarrow 0 \text { in } L^{2}\left(\Omega ; \mathbb{R}^{d \times d}\right) .
\end{gathered}
$$


Since both $\gamma^{0}$ and $\kappa^{0}$ are independent of $\boldsymbol{y}$ we can use Proposition $3.2 i$ to obtain,

$$
\mathcal{U}_{\varepsilon}\left(\gamma^{0}\right) \rightarrow \gamma^{0} \text { in } L^{2}\left(\Omega ; \mathbb{R}^{d \times d}\right)
$$

and

$$
\mathcal{U}_{\varepsilon}\left(\kappa^{0}\right) \rightarrow \kappa^{0} \text { in } L^{2}\left(\Omega ; \mathbb{R}^{d \times d}\right)
$$

Thus, completing the proof.

\section{Conclusions}

We derived effective models for a heterogeneous Cosserat continuum taking into account the Cosserat intrinsic length of the constituents by the method of homogenization and periodic unfolding. In doing so, we provide rigorous proof to the results obtained in [13] by two-scale expansion.

Depending on how the Cosserat intrinsic length scales with respect to the characteristic length of the domain or the chatacteristic length of the periodic cell, we are led to two different effective models. The first effective model is of a classical Cauchy continuum where all the information regarding displacements and rotations at the unit cell are contained in the fourth order stiffness tensor characterizing the material and can be computed by the help of two local problems one of which is related to the curvature-twist. The second effective model is of a Cosserat continuum with two fourth order effective tensors relating the non-symmetric strain to the non-symmetric stress and the curvature-twist to the couple-stress, proving new constitutive laws for Cosserat media.

Additionally, we provide some corrector type results using the averaging operator for each of the effective models. By and large, the results should hold true in the case where one of the materials is a void by adjusting the unfolding and averaging operators, respectively, as in [4].

Acknowledgements The author gratefully acknowledges the funding by the Deutsche Forschungsgemeinschaft (DFG, German Research Foundation) under Germany's Excellence Strategy - The Berlin Mathematics Research Center MATH+ (EXC-2046/1, project ID: 390685689) in project AA2-10. Moreover, the author expresses his gratitude to Prof. Alain Damlamian for his useful remarks and careful critique of the manuscript and to the anonymous referee for the corrections and suggestions. They have, without a doubt, improved its content.

Funding Open Access funding enabled and organized by Projekt DEAL.

Open Access This article is licensed under a Creative Commons Attribution 4.0 International License, which permits use, sharing, adaptation, distribution and reproduction in any medium or format, as long as you give appropriate credit to the original author(s) and the source, provide a link to the Creative Commons licence, and indicate if changes were made. The images or other third party material in this article are included in the article's Creative Commons licence, unless indicated otherwise in a credit line to the material. If material is not included in the article's Creative Commons licence and your intended use is not permitted by statutory regulation or exceeds the permitted use, you will need to obtain permission directly from the copyright holder. To view a copy of this licence, visit http://creativecommons.org/licenses/by/4.0/. 


\section{References}

1. Bensoussan, A., Lions, J.-L., Papanicolaou, G.: Asymptotic analysis for periodic structures. AMS Chelsea Publishing, Providence, RI (1978)

2. Bakhvalov, N., Panasenko, G.: Homogenisation: averaging processes in periodic media: mathematical problems in the mechanics of composite materials. Kluwer Academic Publishers, Amsterdam (1989)

3. Cosserat, E., Cosserat, F.: Théorie des Corps Déformables. Librairie Scientifique A. Hermann et Fils, 6, Rue de la Sorbonne, (1909)

4. Cioranescu, D., Damlamian, A., Donato, P., Griso, G., Zaki, R.: The periodic unfolding method in domains with holes. SIAM J. Math. Anal. 44(2), 718-760 (2012)

5. Cioranescu, D., Damlamian, A., Griso, G.: Éclatement périodique et homogénéisation. C. R. Acad. Sci. Paris Sér. I Math. 335, 99-104 (2002)

6. Cioranescu, D., Damlamian, A., Griso, G.: The periodic unfolding method in homogenization. SIAM J. Math. Anal. 40(4), 1585-1620 (2008)

7. Cioranescu, D., Damlamian, A., Griso, G.: The periodic unfolding method theory and applications to partial differential problems. series in contemporary mathematics, 1st edn. Springer, New York (2018)

8. Damlamian, A.: An elementary introduction to periodic unfolding. Gakuto Int. Series Math. Sci. Appl. 24, 1651-1684 (2005)

9. dell'Isola, F., Corte, A.D., Giorgio, I.: Higher-gradient continua: the legacy of Piola, Mindlin, Sedov and Toupin and some future research perspectives. Math. Mech. Solids 22(4), 852-872 (2017)

10. Eringen, A.C.: Linear theory of micropolar elasticity. J. Math. Mech. 15(6), 909-923 (1966)

11. Eringen, A.C., Suhubi, E.S.: Nonlinear theory of simple microelastic solids-I. Int. J. Eng. Sci. 2(2), 189-203 (1964)

12. Eringen, A.C., Suhubi, E.S.: Nonlinear theory of simple microelastic solids-II. Int. J. Eng. Sci. 2(4), 389-404 (1964)

13. Forest, S., Pradel, F., Sab, K.: Asymptotic analysis of heterogeneous Cosserat media. Int. J. Solids Struct. 38(26-27), 4585-4608 (2001)

14. Forest, S., Sab, K.: Cosserat overall modeling of heterogeneous material. Mech. Res. Commun. 25(4), 449-454 (1998)

15. Forest, S., Sab, K.: Estimating the overall properties of heterogeneous Cosserat materials. Modell. Simul. Mater. Sci. Eng. 7(5), 829-840 (1999)

16. Germain, P.: La méthode des puissances virtuelles en mécanique des milieux continus, I: Théorie du second gradient. J. Mécanique 12(2), 235-274 (1973)

17. Girault, V., Raviart, P.-A.: Finite element methods for Navier-Stokes equations: theory and algorithms, 1st edn. Springer Publishing Company, New York (2011)

18. Guadiello, A., Zappale, E.: Juntion in a thin multidomain for a fourth order problem. Math. Models Methods Appl. Sci. 16(12), 1887-1918 (2006)

19. Guadiello, A., Zappale, E.: A model of joined beams as limit of a 2D plate. J. Elast. 103(2), 205-233 (2011)

20. Hlavacek, I., Hlavacek, M.: On the existence and uniqueness of solution and some variational principles in linear theories of elasticity with couple-stresses. Aplikace Matematiky 14, 387-409 (1969)

21. Hlavácel, I., Necas, J.: On inequalities of Korn's type. i. boundary-value problems for elliptic systems of partial differential equations. Arch. Ration. Mech. Anal. 36, 305-311 (1970)

22. Lakes, R.S.: Size effects and micromechanics of porous solids. J. Mat. Scien. 18, 2572-2581 (1983)

23. Lakes, R.S.: Strongly Cosserat elastic lattice and foam materials for enhanced toughness. Cell. Polym. 12, 17-30 (1993)

24. Lakes, R.S.: On the torsional properties of single osteons. J. Biomech. 28, 1409-1410 (1995)

25. Lions, J.-L.: Quelques méthodes de résolutions des problèmes aux limites non linéaires. GauthierVillars, Paris (1969)

26. Mindlin, R.D., Eshel, N.N.: On first strain-gradient theories in linear elasticity. Int. J. Solids Struct. 4(1), 109-124 (1968)

27. Mindlin, R.D.: Micro-structure in linear elasticity. Arch. Rat. Mech. Anal. 16, 51-78 (1964)

28. Mindlin, R.D.: On the equations of elastic materials with micro-structure. Int. J. Solids Struct. 1(1), 73-78 (1965)

29. Mindlin, R.D., Tiersten, H.F.: Effects of couple-stresses in linear elasticity. Arch. Rat. Mech. Anal. 11, 415-448 (1962) 
30. Mei, C.C., Vernescu, B.: Homogenization methods for multiscale mechanics. World Scientific, Singapore (2010)

31. Necas, J.: Les Méthodes Directes en Théorie des equations Elliptiques, 1st edn. Masson, Paris (1967)

32. Nowacki, W.: The theory of micropolar elasticity. Springer, New York (1972)

33. Onofrei, D., Vernescu, B.: Error estimates for periodic homogenization with non-smooth coefficients. Asym. Anal. 54, 103-123 (2007)

34. Park, H.C., Lakes, R.S.: Cosserat micromechanics of human bone: strain redistribution by a hydrationsensitive constituent. J. Biomech. 19, 385-397 (1986)

35. Pideri, C., Seppecher, P.: A second grade material resulting from the homogenization of a heterogeneous linear elastic medium. Cont. Mech. Thermodyn. 9(5), 241-257 (1997)

36. Rueger, Z., Lakes, R.S.: On the torsional properties of single osteons. Z. Angew. Math. Mech. 68(54), 1-9 (2017)

37. Seppecher, P., Aliberti, J.J., dell'Isola, F.: Linear elastic trusses leading to continua with exotic mechanical interactions. J. Phys.: Conf. Ser. 319, 012018 (2011)

38. Sanchez-Palencia, E.: Non-homogeneous media and vibration theory lecture notes in physics. SpringerVerlag, Berlin Heidelberg (1980)

39. Triantafyllidis, N., Bardenhagen, S.: The influence of scale size on the stability of periodic solids and the role of associated higher order gradient continuum models. J. Mech. Phys. Solids 44(11), 1891-1928 (1996)

40. Toupin, R.: Elastic materials with couple-stresses. Arch. Rat. Mech. Anal. 11(1), 385-414 (1962)

41. Toupin, R.: Theory of elasticity with couple-stress. Arch. Rat. Mech. Anal. 17, 85-112 (1964)

Publisher's Note Springer Nature remains neutral with regard to jurisdictional claims in published maps and institutional affiliations. 